\title{
The Cepheid period-luminosity-metallicity relation based on Gaia DR2 data ${ }^{\star}$
}

\author{
M. A. T. Groenewegen
}

Koninklijke Sterrenwacht van België, Ringlaan 3, 1180 Brussels, Belgium

e-mail: martin.groenewegen@oma.be

Received 23 May 2018 / Accepted 23 July 2018

\begin{abstract}
Aims. We use parallax data from the Gaia second data release (GDR2), combined with parallax data based on HIPPARCOS and HST data, to derive the period-luminosity-metallicity (PLZ) relation for Galactic classical cepheids (CCs) in the $V, K$, and Wesenheit $W V K$ bands.

Methods. An initial sample of $452 \mathrm{CCs}$ are extracted from the literature with spectroscopically derived iron abundances. Reddening values, classifications, pulsation periods, and mean $V$ - and $K$-band magnitudes are taken from the literature. Based on nine CCs with a goodness-of-fit (GOF) statistic smaller than 8 and with an accurate non-Gaia parallax ( $\sigma_{\pi}$ comparable to that in GDR2), a parallax zero-point offset of $-0.049 \pm 0.018$ mas is derived. Selecting a GOF statistic smaller than 8 removes about $40 \%$ of the sample most likely related due to binarity. Excluding first overtone and multi-mode cepheids and applying some other criteria reduces the sample to about 200 stars.

Results. The derived PL(Z) relations depend strongly on the parallax zero-point offset. The slope of the $P L$ relation is found to be different from the relations in the LMC at the $3 \sigma$ level. Fixing the slope to the value found in the LMC leads to a distance modulus (DM) to the LMC of order $18.7 \mathrm{mag}$, larger than the canonical distance. The canonical DM of around 18.5 mag would require a parallax zero-point offset of order -0.1 mas. Given the strong correlation between zero point, period and metallicity dependence of the $P L$ relation, and the parallax zero-point offset there is no evidence for a metallicity term in the PLZ relation.

Conclusions. The GDR2 release does not allow us to improve on the current distance scale based on CCs. The value of and the uncertainty on the parallax zero-point offset leads to uncertainties of order $0.15 \mathrm{mag}$ on the distance scale. The parallax zero-point offset will need to be known at a level of $3 \mu$ as or better to have a 0.01 mag or smaller effect on the zero point of the $P L$ relation and the DM to the LMC.
\end{abstract}

Key words. stars: distances - stars: variables: Cepheids - distance scale - parallaxes

\section{Introduction}

Classical Cepheids (CCs) are considered important standard candles because they are bright and thus the link between the distance scale in the nearby universe and that further out via those galaxies that contain both Cepheids and SNIa (e.g. Riess et al. 2016 for a recent overview on how to get the Hubble constant to $2.4 \%$ precision).

Distances to local CCs may be obtained in several ways, for example through direct determination of the parallax (see below) or main-sequence fitting for Cepheids in clusters (e.g. Feast 1999; Turner 2010 for overviews). In addition, distances to CCs can be obtained from the Baade-Wesselink (BW) method. This method relies on the availability of surface-brightness (SB) relations to link variations in colour to variations in angular diameters and an understanding of the projection $(p-)$ factor, which links radial velocity to pulsational velocity variations. This method is interesting for more distant cepheids where an accurate direct parallax determination is not possible. The most recent works for 70-120 Galactic and about 40 Magellanic Cloud cepheids are by Storm et al. $(2011 \mathrm{a}, \mathrm{b})$ and Groenewegen (2013). These papers also investigated the possible metallicity dependence of the period-luminosity $(P L)$ relation, which is one

* Table 1 is only available at the CDS via anonymous ftp to cdsarc.u-strasbg.fr (130.79.128.5) or via http://cdsarc. u-strasbg.fr/viz-bin/qcat?]/A+A/619/A8 of the remaining possible sources of systematic uncertainties in the application of the $P L$ relation to the distance scale. Although the effect is deemed to be subdominant $(0.5 \%$ on a total uncertainty of $2.4 \%$ in the determination of the Hubble constant, as stated by Riess et al. 2016), estimates in the literature for its actual value and error estimate vary considerably and seem to depend on wavelength (see Storm et al. 2011b; Groenewegen 2013 for references) and a closer investigation is certainly in order in the general framework of "precision cosmology" and a $1 \%$ accurate Hubble constant.

As accurate direct distances to a sizeable number of Galactic Cepheids were unavalaible pre-Gaia the BW method was the only way to investigate this. Both papers agree that the metallicity dependence in the $K$ band is statistically insignificant with the data they had. Storm et al. found a $2 \sigma$ effect in the classical Wesenheit relation based on $V, I[W(V I)=$ $V-2.55(V-I))]$, while Groenewegen found a $2 \sigma$ effect in the $V$ band.

These types of questions can be addressed directly when accurate parallaxes are available for a significant sample of Galactic CCs. The Gaia second data release (GDR2, Gaia Collaboration 2018) extends GDR1 (Gaia Collaboration 2016b,a). The Gaia parallaxes on CCs extend earlier work based on HIPPARCOS parallaxes (ESA 1997; van Leeuwen et al. 2007; van Leeuwen 2007, 2008), and parallel work using the Fine Guidance Sensor (Benedict et al. 2007) and the Wide Field 
Camera 3 (Riess et al. 2014, 2018a; Casertano et al. 2016) on board the Hubble Space Telescope (HST) for about 20 CCs.

In this paper we aim to investigate the $P L$ relation and its possible metallicity dependence based on a sample of Galactic cepheids available in the Gaia DR2. The paper is structured as follows. In Sect. 2 the collection of photometric, reddening, metallicity, and other data from the sample is described. Section 3 describes the data taken from GDR2, and compares periods and classifications from the literature with those provided in GDR2. The method used in the analysis in described in Sect. 4, and tested with simulations in Sect. 5. Section 6 presents the results which are summarised and discussed in Sect. 7.

\section{Pre-Gaia DR2 preparation}

The preparation for this paper started with the collection from the literature of all CCs with individually determined accurate iron abundances from high-resolution spectroscopy (see below for detailed references). This resulted in a sample of 452 stars, and the data described below are listed in Table 1.

To perform the study on the metallicity dependence of the $P L$ relation, the following data are required: the classification (Cepheids can be fundamental mode (FU) pulsators, first overtone (FO) or second overtone (SO) pulsators, or doublemode (DM) pulsators); the pulsation period; magnitudes (in this paper we concentrate on $V$, and the near-IR magnitudes $J H K$ ); the reddening $E(B-V)$ to de-redden the photometry; the metallicity (synonymous here with the iron abundance $[\mathrm{Fe} / \mathrm{H}]$ ); and the parallax.

Mean $V$ magnitudes are taken mainly from Mel'nik et al. (2015). This reference provides standard Johnson $V$ magnitudes for a sample of 674 cepheids, the latest extension of the collection of optical photometry following Berdnikov et al. (2000). Only 28 of the stars are not listed there. The footnote to Table 1 contains details on the photometry that was used. In some cases mean magnitudes were derived by fitting Fourier series to time series data using the PERIOD04 software (Lenz \& Breger 2005). From a comparison of the mean magnitudes quoted in different sources, an error in the mean magnitude of $0.008 \mathrm{mag}$ is adopted.

The near-IR (NIR) photometry is more heterogeneous as it comes from a variety of sources, using different photometric systems and ranges from intensity-mean magnitudes from well-sampled light curves to single-epoch photometry in some cases. In order of preference, mean magnitudes are taken from Monson \& Pierce (2011), converted to the 2MASS system based on the transformation equations in their Table 1; SAAO-based photometry (mainly Laney \& Stobie 1992, and Laney, priv. comm., as quoted in Genovali et al. 2014 and Feast et al. 2008), converted to the 2MASS system based on the transformation equations in Koen et al. (2007); and CIT-based photometry from Welch et al. (1984) and Barnes et al. (1997), converted to the 2MASS system based on the transformation equations in Monson \& Pierce (2011). For the remaining sources the median was taken of the available single-epoch data available in McGonegal et al. (1983), Welch et al. (1984), Schechter et al. (1992), DENIS ( $J K$ data transformed to the 2MASS system using Carpenter 2001), and 2MASS.

For the data by Monson \& Pierce (2011) and the SAAObased data an error in the mean magnitude of 0.008 mag was assumed. For the mean magnitudes from CIT-based data an error of $0.01 \mathrm{mag}$ was assumed as they generally appear to be of slightly lower quality. For the photometry based on median filtering of multiple observations an error of 0.025 mag was assumed. If only a single-epoch 2MASS observation was available a typical error of 0.025 mag was assumed, unless the quality flag was not AAA, in which case a typical error of 0.25 mag was assumed.

A special case is Polaris. The 2MASS magnitude is highly uncertain $(K=0.456 \pm 0.248 \mathrm{mag})$. The COBE-DIRBE flux at 1.25 and $2.2 \mu \mathrm{m}$ was taken (Smith et al. 2004) and converted to magnitudes using the $2 \mathrm{MASS}$ zero points (ZPs). Including error bars in the flux and in the ZPs we arrives at $J=0.941 \pm 0.031$ and $K=0.652 \pm 0.028 \mathrm{mag}$. As Polaris is hardly variable, this is essentially an estimate of the mean intensity. This value is consistent with the older photometry by Gehrz \& Hackwell (1974a). Taking the ZP of that system (Gehrz et al. 1974b), and converting the flux back to a 2 MASS magnitude we arrive at an estimate $K=0.64 \pm 0.10 \mathrm{mag}$.

Reddening values, $E(B-V)$, and the error therein are primarily taken from the compilation in Fernie et al. (1995) ${ }^{1}$ with a scaling factor as indicated below. Only about 50 stars are not listed there.

Tammann et al. (2003) suggested scaling the values in Fernie et al. (1995) by a factor of 0.951 to have consistency between the values listed there and those derived from a periodcolour relation. Fouqué et al. (2007) also discussed reddening and adopted the reddening from Laney \& Caldwell (2007) based on BVI photometry, which is also adopted here. Fouqué et al. (2007) find a scaling factor of $0.952 \pm 0.010$ with respect to the reddenings listed in Fernie et al. (1995). The stars in Fouqué et al. (2007) were compared to those in Laney \& Caldwell (2007). Thirty-nine are in overlap, of which 27 have $E(B-V)>0.2$ mag. The ratio of the reddenings lies between $0.91-1.18$ and both the median and mean ratio are 1.00 with a dispersion of 0.03 . Comparing Fouqué et al. (2007) to Fernie et al. (1995) there are 127 stars with $E(B-V)>0.2$ mag in overlap, with a range in ratios of $0.80-1.42$ with mean and median of $0.93-0.94$ and dispersion 0.05. Similarly, comparing Tammann et al. (2003) to Fernie et al. (1995) there are 184 stars with $E(B-V)>0.2$ mag in overlap, with a range in ratios of $0.78-1.16$ with mean and median of $0.94-0.95$ and dispersion 0.05 .

In order of preference, reddenings were taken from Fernie et al. (1995) scaled by a factor of 0.94; Acharova et al. (2012) without scaling, Luck \& Lambert (2011) scaled by 0.99, Caldwell \& Coulson (1987) scaled by 0.987, Kashuba et al. (2016) scaled by 0.94, Martin et al. (2015) scaled by 0.97 , and Sziládi et al. (2007) scaled by 0.92 . For eight stars no reddening appears to have been published, and these were estimated from several 3D reddening models (Marshall et al. 2006; Drimmel et al. 2003; Arenou et al. 1992) using the parallax from GDR2 (see Groenewegen 2008 for details).

The error in $E(B-V)$ is taken from Fernie et al. (1995) or from the spread among the different $3 \mathrm{D}$ reddening estimates. Otherwise it is assumed to be $0.1 E(B-V)$. The extinction in the visual is assumed to be $A_{V}=3.1 E(B-V)$, and extinction ratios $A_{J} / A_{V}, A_{H} / A_{V}$, and $A_{K} / A_{V}$ of $0.276,0.176$, and 0.118 , respectively, have been adopted.

The iron abundances are taken from several sources and put on the same scale. The main source is the compilation by Genovali et al. (2014), which has data for 434 stars when combined with Genovali et al. (2015). They compared iron abundances from different literature sources and re-scaled all data to a uniform scale. Column 12 in Table 1 lists the iron abundance, if available, from Genovali et al. (2014) or from the follow-up work in Genovali et al. (2015).

http://www . astro. utoronto. ca/DDO/research/cepheids/ table_colourexcess.html 
Another large compilation is that in Ngeow (2012), which has 329 stars in common with Genovali et al. (2014, 2015). The average difference (in the sense Ngeow - Genovali et al. $)=+0.02 \pm 0.06$ dex. Ngeow works on the system by Luck, Lambert, and coworkers, and therefore a direct comparison is made to the iron abundances in Luck \& Lambert (2011). There are 318 stars in common with Genovali et al. (2014, 2015). The average difference (in the sense Luck \& Lambert - Genovali et al. $)=+0.03 \pm 0.05$ dex. Column 14 in Table 1 lists the iron abundance, if available, from Ngeow (2012) or Luck \& Lambert (2011) without further correction.

Some other catalogues were also considered. The large compilation by Acharova et al. (2012) has 277 stars in common with Genovali et al. $(2014,2015)$. The average difference (in the sense Acharova et al. - Genovali et al.) is $-0.055 \pm 0.08$ dex. Sziládi et al. (2007) has 14 stars in common with Genovali et al. (2014). The average difference (in the sense Sziládi et al. Genovali et al.) is $-0.032 \pm 0.07$ dex. Martin et al. (2015) has 22 stars in common with Genovali et al. (2014). The average difference (in the sense Martin et al. - Genovali et al.) is $-0.03 \pm 0.07$ dex. Column 16 in Table 1 lists the iron abundance, if available, from these three references, with offsets applied. In the analysis below the value in Col. 12 is preferred over that in Col. 14, which is preferred over that in Col. 16. Based on the comparison between datasets and the scatter between different measurements, an error of $0.08 \mathrm{dex}$ in $[\mathrm{Fe} / \mathrm{H}]$ is assumed.

Regarding the variability type and pulsation period the Variable Star indeX catalogue (VSX; Watson et al. 2006) was the main source of information, but other sources were also consulted (Berdnikov et al. 2000; Klagyivik \& Szabados 2009; Luck \& Lambert 2011; Ngeow 2012; Genovali et al. 2014; Mel'nik et al. 2015). Periods agree typically to a high degree, of order $4 \times 10^{-4} \mathrm{P}$ or better. Pulsation types are sometimes less certain. This can be related to the FU or FO classification, or even the classification as CC. The star V473 Lyr is assumed to be a SO CC (Molnár et al. 2017).

The stars BC Aql, TX Del, AU Peg, and SU Sct are classified as (likely) Type-II Cepheids (T2Cs). QQ Per is also marked as an uncertain CC (indicated by the "?") and has been classified as a T2C as well. Two stars have a very different classification. The star EK Del is classified as a possible Above the Horizontal Branch $(\mathrm{AHB})$ star. Its metallicity of $[\mathrm{Fe} / \mathrm{H}]=-1.57 \mathrm{dex}$ is by far the lowest among the 452 objects and seems more closely related to that of RR Lyrae. The object V1359 Aql is classified as "ROT", i.e. a spotted star whose variability is due to rotation, with a period of 96.3 days. These stars were kept in the sample, anticipating that the GDR2 would also contain classifications for many variables (see next section and Table 2).

The total number of stars that is potentially used for the analysis of the PLZ relation is 426; the starting sample of 452 listed in Table 1, minus 2 targets not listed in GDR2 (see next section), minus 6 stars almost certainly not CCs, and minus 18 stars that are SO or DM Cepheids that were also a priori excluded.

\section{Gaia DR2 data}

The data was obtained by querying the various tables through VizieR. The list of objects was cross-matched with the gaiadr2.gaia_source table using a radius of $1.2^{\prime \prime}$. The largest differences were for CE Cas A (at 0.9") and Polaris (at 0.6"). The other sources were matched to within $0.3^{\prime \prime}$ or better. Two sources, V340 Nor and IY Cep, were not found (even when a larger search radius was used), and they appear to be missing from GDR2.
Table 2. Comparison of pulsation types and modes.

\begin{tabular}{|c|c|c|c|c|}
\hline Name & $\begin{array}{r}\text { Literature } \\
\text { (Table 1) }\end{array}$ & $\begin{array}{r}\text { GDR2 } \\
\text { SOS }\end{array}$ & $\begin{array}{l}\text { GDR2 } \\
\text { nTran }\end{array}$ & Remarks \\
\hline BG Cru & DCEPS & DCEP-FU & CEP & \\
\hline CI Per & DCEP? & DCEP-FO & T2CEP & \\
\hline CR Cep & DCEP? & DCEP-FU & CEP & \\
\hline CY Aur & DCEP & DCEP-FO & CEP & Period incorrect \\
\hline DK Vel & DCEP & DCEP-FO & CEP & \\
\hline FM Aql & DCEP & DCEP-FO & CEP & Period incorrect \\
\hline FO Cas & DCEP & T2CEP & T2CEP & \\
\hline GH Car & DCEPS & DCEP-FU & CEP & \\
\hline IT Car & DCEPS & DCEP-FU & CEP & \\
\hline MY Pup & DCEPS & DCEP-FU & CEP & \\
\hline NT Pup & DCEP & T2CEP & T2CEP & \\
\hline RS Ori & DCEP & DCEP-FO & CEP & Period incorrect \\
\hline RW Cam & DCEP & T2CEP & CEP & \\
\hline TT Aql & DCEP & DCEP-FO & CEP & Period incorrect \\
\hline TU Cas & DCEP(B) & DCEP-FU & CEP & \\
\hline TX Del & CWB: & T2CEP & CEP & \\
\hline V1334 Cyg & DCEPS & DCEP-FU & CEP & \\
\hline V350 Sgr & DCEP & DCEP-FO & CEP & Period incorrect \\
\hline V378 Cen & DCEPS & DCEP-FU & CEP & \\
\hline V482 Sco & DCEP & DCEP-FO & CEP & Period incorrect \\
\hline V500 Sco & DCEP & T2CEP & CEP & Period incorrect \\
\hline V636 Cas & DCEPS & DCEP-FU & CEP & \\
\hline V659 Cen & DCEPS & DCEP-FU & CEP & \\
\hline V924 Cyg & DCEPS & DCEP-FU & CEP & \\
\hline X Lac & DCEPS & DCEP-FU & CEP & \\
\hline Y Oph & DCEP? & DCEP-FU & CEP & \\
\hline Y Car & DCEP(B) & DCEP-FO & CEP & \\
\hline GZ Car & DCEP(B) & DCEP-FU & CEP & \\
\hline BK Cen & DCEP(B) & DCEP-FU & CEP & \\
\hline V458 Sct & DCEP(B) & DCEP-FU & CEP & Period incorrect \\
\hline $\mathrm{U} \operatorname{Tr} \mathrm{A}$ & $\operatorname{DCEP}(\mathrm{B})$ & DCEP-FU & CEP & \\
\hline V493 Aql & DCEP & MULTI & CEP & Period incorrect \\
\hline V526 Aql & DCEP & T2CEP & T2CEP & Period incorrect \\
\hline CO Aur & DCEPS(B) & DCEP-FO & CEP & \\
\hline AC Cam & DCEP & MULTI & - & \\
\hline FW Cas & DCEP & MULTI & - & \\
\hline HK Cas & DCEP & DCEP-FO & ACEP & \\
\hline EK Del & AHB1: & DCEP-FU & ACEP & \\
\hline FQ Lac & CEP:? & DCEP-FU & T2CEP & \\
\hline BE Mon & DCEP & DCEP-FO & CEP & \\
\hline QQ Per & CEP? & T2CEP & T2CEP & \\
\hline CR Ser & DCEP & DCEP-FO & CEP & Period incorrect \\
\hline V1954 Sgr & DCEP & DCEP-FO & CEP & Period incorrect \\
\hline
\end{tabular}

Notes. Pulsation type and mode from the literature (Col. 2, as listed in Table 1) and from the SOS module (Col. 3). In Col. 3 the classification from the nTransits:2+ classification scheme.

From the source table the following parameters were retrieved:

- The unique source identifier source_id for querying other tables (see below);

- The parallax $(\pi)$ and parallax_error $\left(\sigma_{\pi}\right)$ (both in mas);

- Parameters describing the quality of the astrometric fit, in particular,

1) the goodness-of-fit (GOF) statistic, astrometric_ gof_al, of the astrometric solution for the source in the along-scan direction. For good fits it should approximately follow a normal distribution with zero mean value and unit standard deviation; 

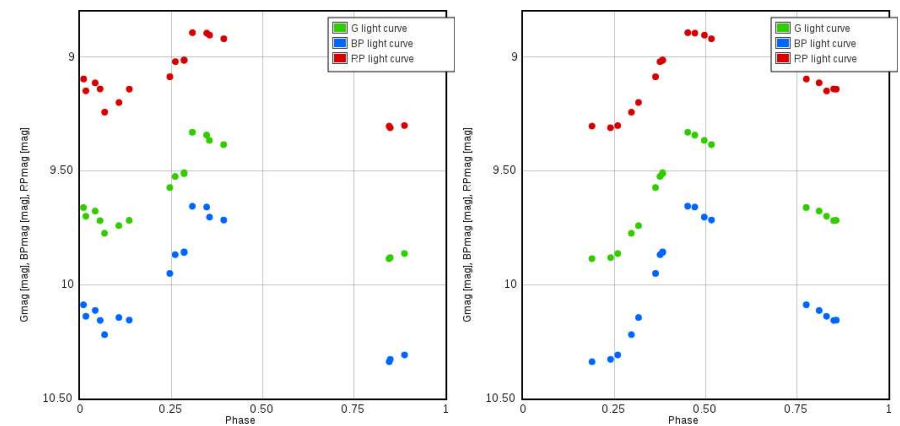

Fig. 1. Example of the phased light curve for AD Gem in the Gaia $G, B p$, and $R p$ bands fitted with the period as given by the SOS (1.895 days, left) and the correct period (3.788 days, right). The plots were made using the tools available through VizieR.

2) the astrometric_excess_noise, $\epsilon_{i}$, which is quadratically added to the assumed observational noise in each observation in order to statistically match the residuals in the astrometric solution and the astrometric excess_noise_sig, $D$, the significance of $\epsilon_{i}$, where "A value $D>2$ indicates that the given $\epsilon_{i}$ is probably significant" .

We note that the catalogued values for these parameters have not been corrected for the "DOF bug", as discussed in Appendix A in Lindegren et al. (2018).

The GOF parameter was also given in the HIPPARCOS data releases, but was not listed in GDR1. The excess noise parameter and its significance were parameters introduced in GDR1.

GDR2 provides additional information, in particular regarding variability (see Holl et al. 2018). Two types of classification and analysis are available. The first is based on at least two transits, the nTransits:2+ classifier, which gives a best_class_name, and a best_class_score, a number between 0 and 1 , indicating the confidence of the classification. More useful information is available when more transit data is available and the objects are passed through Specific Objects Studies (SOS) modules. In particular a total of 9575 objects have been classified as a Cepheid by the SOS module on Cepheids and RR Lyrae (Clementini et al. 2018). Based on the source_id the vari_cepheid table was queried to return the following:

- The type_best_classification, which can be DCEP, T2CEP, and ACEP respectively for Classical Cepheids, Type-II Cepheids, and Anomalous Cepheids;

- The mode_best_classification, which can be FUNDAMENTAL, FIRST OVERTONE, or MULTI;

- The pulsation period with error (in the case of a MULTI classification two periods are given);

- The metallicity of the star derived from the Fourier parameters of the light curve, and its error.

Of the sample 257 are classified as FU pulsators, 43 as FO, 6 as multi-mode, and 8 as T2C by the SOS module. The nTransits: $2+$ classifier lists 5 Anomalous Cepheids, 50 T2C, $300 \mathrm{CCs}$, and $7 \mathrm{Mira} /$ Semi-regular pulsators.

Based on the data in GDR2 some checks were performed against the data that was prepared pre-Gaia. For 279 stars the periods derived by the SOS module compare well to the value

2 See the Gaia Data Release 2 documentation available at https://gea.esac.esa.int/archive/documentation/GDR2/pdf/ GaiaDR2_documentation_1.1.pdf for a description of these parameters.

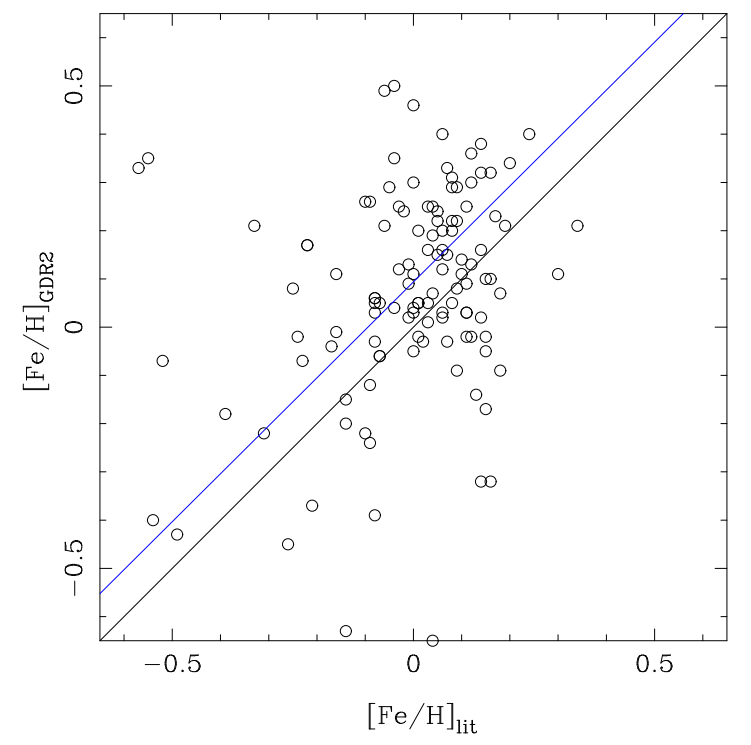

Fig. 2. Comparison of the $[\mathrm{Fe} / \mathrm{H}]$ abundance from the literature to that of the SOS. The black line is the one-to-one relation. The blue line is the bi-sector fit to the data.

in the literature (in Table 1), with a relative precision better than $0.5 \%$ and a median value $|\Delta P| / P=1.3 \times 10^{-4}$. Then there is a jump, and for 35 stars the periods are significantly different with $|\Delta P| / P>0.06$. The most extreme example is V500 Sco with a true period of 9.3 and a derived period of 175.6 days. Other periods differ by a near integer number, for example $\mathrm{T}$ Vul (true period of 4.435 and a derived period of 2.217 days) or XX Car (true period of 15.716 and a derived period of 31.449 days). All 35 cases were inspected by folding the Gaia light curve with the period in the literature; the literature period always fits the Gaia light curve better (see Fig. 1 for an example).

In the present sample about $11 \%$ of the Cepheids have been assigned an incorrect period. As the paper describing the SOS Cepheid and RR Lyrae module used for GDR2 (Clementini et al. 2018) does not contain any information about the period validation for Cepheids it is unclear how representative this fraction is.

Of interest is also the classification of the objects, and their pulsation mode. Of the 314 stars in the sample analysed by the SOS, 271 classifications agree with the value in the literature. The other 43 cases are listed in Table 2. The most common difference is between the FU and FO pulsations. As noted above some periods are incorrect, and this is indicated as it might have influenced the classification as well.

Interestingly, the SOS module also provides an iron abundance estimate based on the shape of the $G$-band light curve for 120 objects in the sample. Figure 2 compares the adopted $[\mathrm{Fe} / \mathrm{H}]$ abundance from the literature listed in Table 1 with the value provided in GDR2. There is a correlation, but with a lot of scatter. A bi-sector fit gives a slope of 0.99 and a zero point of 0.09 . The scatter around this relation is $0.26 \mathrm{dex}$, comparable to the quoted uncertainty of $0.22-0.24 \mathrm{dex}$ (which includes a systematic error of 0.2 dex; see Clementini et al. 2018). This justifies the choice of considering only Cepheids with spectroscopic abundance determinations.

Table 3 lists the CCs with accurate external parallaxes (i.e. non-Gaia, non-HIPPARCOS) and compares them to Gaia DR2, Gaia DR1, and HIPPARCOS parallaxes. The external parallaxes are mostly based on HST (Benedict et al. 2007; Riess et al. 2014, 2018a; Casertano et al. 2016). Out of interest, the parallax of Polaris B is also listed, with the results from GDR2 
M. A. T. Groenewegen et al.: The Cepheid period-luminosity-metallicity relation based on Gaia DR2 data

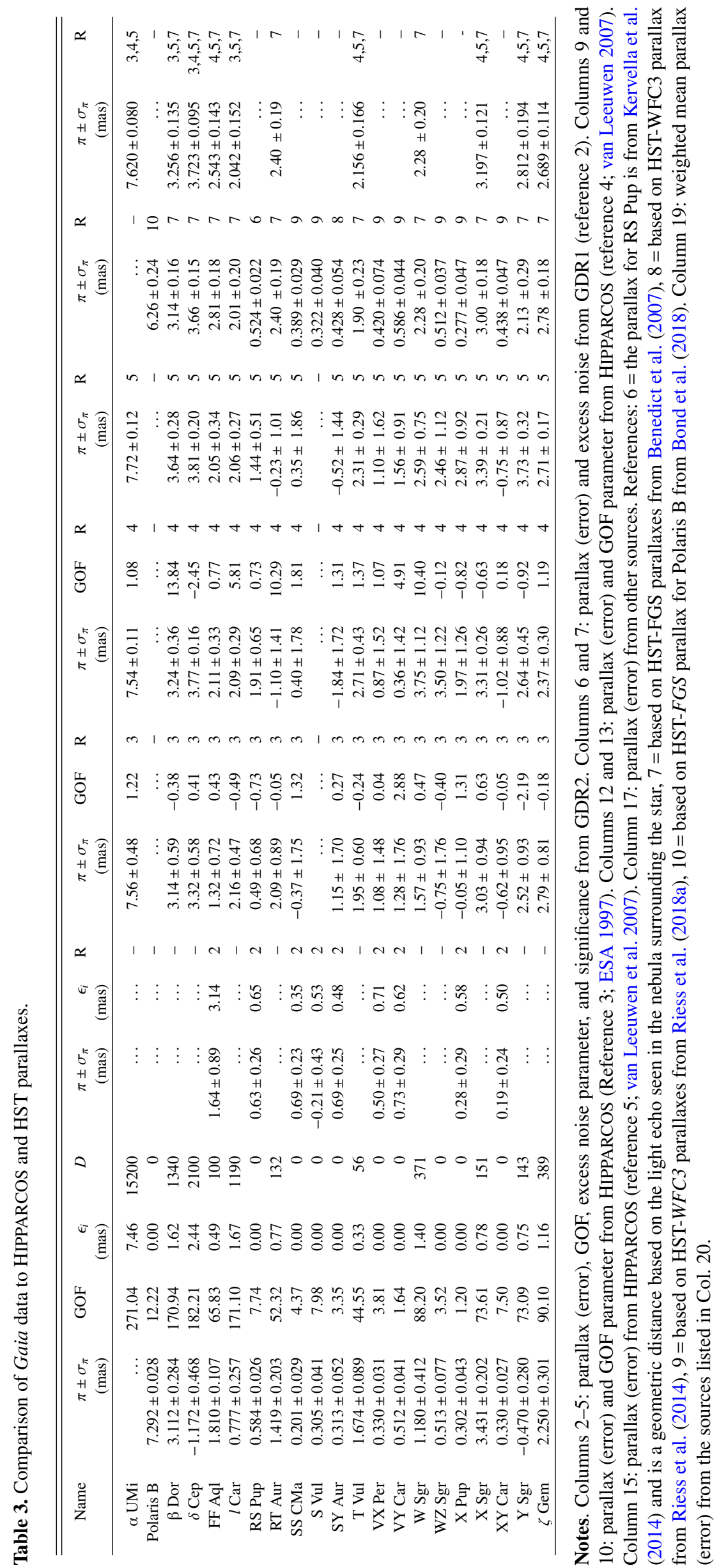


and the parallax as recently determined by Bond et al. (2018). Polaris B is thought to be physically related to $\alpha$ UMi (see Anderson 2018 and discussion therein). Another parallax estimate of $10.1 \pm 0.2$ mas exists for Polaris A (Turner et al. 2013) based on the claimed membership of a cluster, but this result has been disputed (van Leeuwen 2013). The photometric parallax of Polaris predicted by the $P L$ relation derived in the present paper is discussed in Appendix B.

What is remarkable in Table 3 is that many of the well-known Cepheids have very poor solutions with very large GOF and excess noise values. There almost seems to be a dichotomy with Cepheids with zero excess noise to have a GOF smaller than 8 . Figure 3 shows the relation between these two parameters for the entire sample, and the distribution over the GOF parameter. We note that this distribution is not a Gaussian with mean zero and unit variance, but it is due to the fact that this parameter was not updated after discovery of the DOF bug (Lindegren et al. 2018).

The bottom panel in Fig. 3 shows the distribution over the GOF parameter when it is recomputed multiplying the astrometric_chi2_al statistic by a constant for all sources and to force a peak in the histogram near zero. The factor used is 0.7, which is roughly consistent with the information provided in Appendix A in Lindegren et al. (2018).

All nine stars with a GOF smaller than 8 have an accurate external parallax ( $\sigma_{\pi}$ comparable to that in GDR2). The weighted mean difference (in the sense GDR2-external parallax) is $-0.049 \pm 0.018$ mas. It is tempting to relate this to the parallax zero-point offset observed for QSO ( -0.029 mas, Lindegren et al. 2018), based on RGB stars from Kepler and APOGEE data (about -0.053 mas; Zinn et al. 2018), eclipsing binaries $(-0.082 \pm 0.033$ mas; Stassun \& Torres 2018), a sample of $50 \mathrm{CCs}(-0.046 \pm 0.013$ mas; Riess et al. 2018b), RR Lyrae stars ( $\sim-0.056$ mas; Muraveva et al. 2018), and the value of $-0.0319 \pm 0.0008$ mas mentioned for Cepheids in the GDR2 catalogue validation paper (Arenou et al. 2018).

It should be noted that the assumption of a constant parallax zero-point offset is an oversimplification. Lindegren et al. (2018) already show that there are correlations with position on the sky, and trends with magnitude and colour (their Figs. 7, 12, 13).

Binarity is common among Cepheids and has not been discussed so far. Binarity is not considered in solving for the astrometric parameters in GDR2. If binarity has an effect it would express itself in a poor fit when only solving for position, proper motion, and parallax. As noted in Appendix A in Lindegren et al. (2018) the statistical quantities astrometric_chi2_al and the GOF statistic have not been corrected for the DOF bug. The GOF statistic is expected to follow a normal distribution around zero with unit variance, but for the current sample it roughly follows a normal distribution which peaks near 4 and with a clear excess of stars with a GOF $>8$. The models in Appendix A show that selecting on GOF $<8$ removes $40 \%$ of the sample. Many of those are known binaries. Riess et al. (2018b) identify three outliers in their sample of $50 \mathrm{CCs}$, based on the location in a simple $\sigma_{\pi}$ versus $\pi$ plot: SV Per, RW Cam, and RY Vel. The GOF statistic of these objects is 84,85 , and 38 , respectively, and SV Per and RW Cam show close companions in their HST images. Other known binaries have large GOF statistics and are therefore excluded from the analysis: V1334 Cyg $(\mathrm{GOF}=37)$ from Evans (2000) and Gallenne et al. (2013); AX Cir (GOF = 387), KN Cen $(\mathrm{GOF}=8.5), \mathrm{SY}$ Nor $(\mathrm{GOF}=13), \mathrm{AW}$ Per $(\mathrm{GOF}=8.6)$, and SV Per $(\mathrm{GOF}=85)$ from Evans $(1994)$, and R Cru $(\mathrm{GOF}=92)$ and $S$ Mus $(\mathrm{GOF}=60)$ from Evans et al. (2016). It appears that selecting a GOF $<8$ is an effective way of removing binaries from
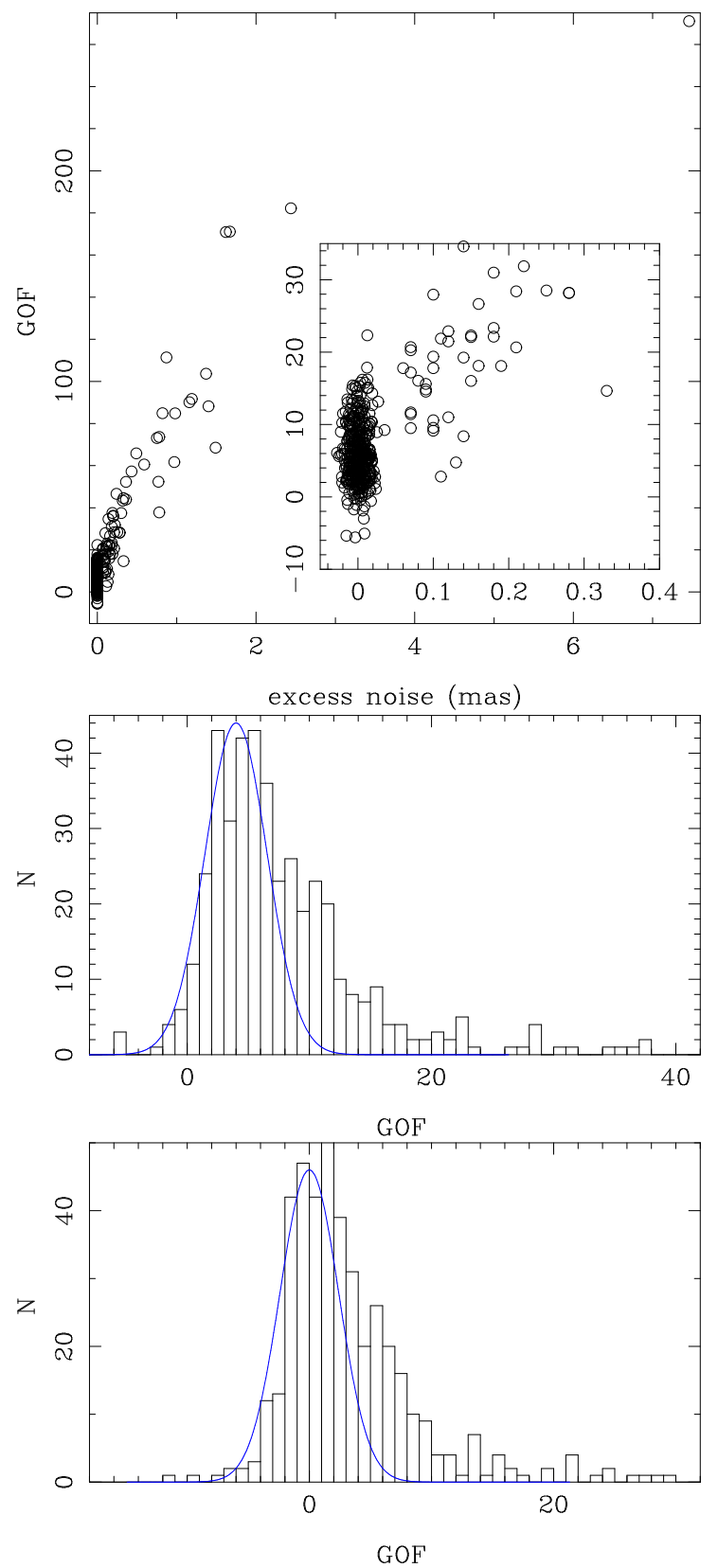

Fig. 3. Goodness-of-fit (GOF) parameter plotted against the excess noise, with the inset showing a zoomed-in version. At the extreme end is Polaris with $\epsilon_{i}=7.5$ and GOF $=270$ (top panel). For clarity a randomly Gaussian distributed number is added to the excess noise when it is below 0.02 mas in the inset. In the middle panel the histogram of the GOF parameter is shown with a Gaussian distribution (with mean 4.0 and $\sigma=1.8$ ) overplotted in blue. In the bottom panel the GOF statistic has been recomputed by multiplying the astrometric_chi2_al statistic by 0.7 for all sources to force a peak in the histogram at about zero. The $\sigma$ of the Gaussian is 1.7. The abscissa has been shifted by ten units compared to the plot in the middle panel.

GDR2 data, and suggests that the results are not systematically influenced by binarity in the remaining sample.

\section{Analysis}

The fundamental equation between parallax, apparent, and absolute magnitude is

$\pi=100 \times 10^{0.2(M-m)}$, 
Table 4. Simulations of the $P L$ relations of the form $M=\alpha+\beta \log P$ with input values $\alpha=-2.50$ and $\beta=-3.30$.

\begin{tabular}{|c|c|c|c|c|}
\hline \# & $\alpha$ & $\beta$ & $N$ & Remarks \\
\hline \multicolumn{5}{|c|}{ Using exact distance } \\
\hline 1 & $-2.502 \pm 0.006$ & $-3.296 \pm 0.007$ & 426 & $1 \%$ error on distance \\
\hline 2 & $-2.502 \pm 0.009$ & $-3.296 \pm 0.011$ & 426 & $2 \%$ error on distance \\
\hline 3 & $-2.502 \pm 0.018$ & $-3.296 \pm 0.020$ & 426 & $5 \%$ error on distance \\
\hline 4 & $-2.504 \pm 0.037$ & $-3.294 \pm 0.041$ & 426 & $10 \%$ error on distance \\
\hline \multicolumn{5}{|c|}{ With parallax error } \\
\hline 5 & $-2.499 \pm 0.006$ & $-3.297 \pm 0.007$ & 426 & $1 \%$ error on distance \\
\hline 6 & $-2.500 \pm 0.009$ & $-3.294 \pm 0.011$ & 426 & $2 \%$ error on distance \\
\hline 7 & $-2.496 \pm 0.018$ & $-3.293 \pm 0.020$ & 426 & $5 \%$ error on distance \\
\hline 8 & $-2.488 \pm 0.037$ & $-3.294 \pm 0.041$ & 426 & $10 \%$ error on distance \\
\hline 9 & $-2.521 \pm 0.021$ & $-3.267 \pm 0.022$ & 426 & Parallax error based on data \\
\hline 10 & $-2.518 \pm 0.027$ & $-3.268 \pm 0.030$ & 426 & Error based on data, another random seed \\
\hline 11 & $-2.514 \pm 0.023$ & $-3.273 \pm 0.025$ & 426 & Error based on data, another random seed \\
\hline 12 & $-2.511 \pm 0.021$ & $-3.276 \pm 0.023$ & 361 & Error based on data, $\pi>0, \sigma_{\pi} / \pi<0.2$ \\
\hline 13 & $-2.514 \pm 0.024$ & $-3.273 \pm 0.027$ & 218 & Error based on data, $\pi>0, \sigma_{\pi} / \pi<0.1$ \\
\hline 14 & $-2.557 \pm 0.022$ & $-3.295 \pm 0.024$ & 426 & Error based on data, ZPoff $=-0.029$ mas, not in analysis \\
\hline 15 & $-2.577 \pm 0.022$ & $-3.312 \pm 0.024$ & 426 & Error based on data, ZPoff $=-0.046$ mas, not in analysis \\
\hline 16 & $-2.521 \pm 0.022$ & $-3.267 \pm 0.023$ & 426 & Error based on data, ZPoff $=-0.046$ mas, -0.046 in analysis \\
\hline 17 & $-2.541 \pm 0.022$ & $-3.284 \pm 0.023$ & 426 & Error based on data, ZPoff $=-0.046$ mas, -0.029 in analysis \\
\hline 18 & $-2.565 \pm 0.023$ & $-3.319 \pm 0.025$ & 310 & Error based on data, ZPoff $=-0.046$ mas, not in analysis, $\pi>0, \sigma_{\pi} / \pi<0.2$ \\
\hline 19 & $-2.562 \pm 0.023$ & $-3.308 \pm 0.024$ & 189 & Error based on data, ZPoff $=-0.046$ mas, not in analysis, $\pi>0, \sigma_{\pi} / \pi<0.1$ \\
\hline 20 & $-2.572 \pm 0.025$ & $-3.271 \pm 0.027$ & 76 & Error based on data, ZPoff $=-0.046$ mas, not in analysis, $\pi>0, \sigma_{\pi} / \pi<0.05$ \\
\hline 21 & $-2.542 \pm 0.040$ & $-3.276 \pm 0.038$ & 13 & Error based on data, ZPoff $=-0.046$ mas, not in analysis, $\pi>0, \sigma_{\pi} / \pi<0.02$ \\
\hline 22 & $-2.575 \pm 0.026$ & $-3.273 \pm 0.028$ & 126 & Error based on data, ZPoff $=-0.046$ mas, not in analysis, $\pi>0.5$ mas \\
\hline 23 & $-2.570 \pm 0.030$ & $-3.257 \pm 0.033$ & 50 & Error based on data, ZPoff $=-0.046$ mas, not in analysis, $\pi>1.0$ mas \\
\hline 24 & $-2.539 \pm 0.035$ & $-3.279 \pm 0.035$ & 20 & Error based on data, ZPoff $=-0.046$ mas, not in analysis, $\pi>1.5$ mas \\
\hline 25 & $-2.515 \pm 0.033$ & $-3.288 \pm 0.039$ & 205 & As $(9)$, for realistic sample size and $\delta_{\mathrm{PL}}=0 \mathrm{mag}$ \\
\hline 26 & $-2.520 \pm 0.058$ & $-3.289 \pm 0.066$ & 205 & As (9), for realistic sample size and $\delta_{\mathrm{PL}}=0.066 \mathrm{mag}$ \\
\hline
\end{tabular}

Notes. $N$ is the number of stars in the solution.

where $\pi$ is the parallax in mas, and $m$ the dereddened apparent magnitude. The absolute magnitude $M$ is parameterised as

$M=\alpha+\beta \log P+\gamma[\mathrm{Fe} / \mathrm{H}]$

and the aim is to derive the coefficients $\alpha, \beta$, and $\gamma$.

Feast \& Catchpole (1997) had a similar aim in mind using HIPPARCOS data. The accuracy of the HIPPARCOS data was such that only the simpler problem with $\gamma=0$ and known slope $\beta$ (from Cepheids in the Large Magellanic Cloud, LMC) could be tackled. In that case the problem is simplified to $10^{0.2 \alpha}=0.01 \times$ $\pi \times 10^{0.2(m-\beta \log P)}$. The zero point of the $P L$ relation was found by taking the weighted mean of the term on the right-hand side over all 223 Cepheids available to them, and then calculating 5 times the logarithmic value. For an assumed slope of -2.81 in the $V$ band they derived $\alpha=-1.43 \pm 0.10 \mathrm{mag}$. Using the revised HIPPARCOS parallaxes van Leeuwen et al. (2007) found $\alpha=-2.47 \pm 0.03 \mathrm{mag}$ (the weighted mean of the three values in their Table 6) for fixed $\beta=-3.26$ in the $K$ band, see Table 5 .

In the present paper, in principle, we want to solve for all coefficients and therefore the non-linear problem of fitting Eq. (1) is solved directly using the Levenberg-Marquardt algorithm (as implemented in Fortran in Numerical Recipes, Press et al. 1992).

An important advantage of using Eq. (1) in this form is that no selection on positive parallaxes or relative parallax error is required, and therefore the results are not subject to Lutz-Kelker bias (Lutz \& Kelker 1973; see the discussion in Feast \& Catchpole 1997; Koen \& Laney 1998; Lanoix et al. 1999). It is also one of the methods, known as astrometry-based luminosity (ABL), used in Gaia Collaboration (2017) to analyse GDR1 data (also see Luri et al. 2018). Another advantage is that the errors in the parallax can be assumed to be symmetric and Gaussian distributed.

Monte Carlo simulations are carried out for an improved understanding of the results. The basic data of the Cepheids (Table 1) are read in, together with the parameters of the Gaia DR2 (or the external parallax data). Periods of FO pulsators (type DCEPS) are fundamentalised using $P_{0}=P_{1} /(0.716-$ $0.027 \log P_{1}$ ) following Feast \& Catchpole (1997). Then,

- A new parallax is drawn from a Gaussian with the adopted mean and error. A parallax zero-point offset may be applied;

- A new period is drawn from a Gaussian with the mean input period and an error equal to $1.3 \times 10^{-4} \mathrm{P}$;

- A new $[\mathrm{Fe} / \mathrm{H}]$ is drawn from a Gaussian with the mean input iron abundance and an error of $0.08 \mathrm{dex}$;

- A new reddening is drawn from a Gaussian with the mean and error from the input. Negative reddenings are set to zero;

- The input $V, J, H, K$ magnitudes are dereddened (see Sect. 2 for details);

- New $V, J, H, K$ magnitudes are drawn from Gaussians using the dereddened magnitudes and assumed error bars (see Sect. 2 for details);

- The NIR magnitudes are transformed to the 2MASS system if needed;

- To take into account the intrinsic width in the instability strip (see Feast \& Catchpole 1997) the value of $(M-m)$ is increased by a value drawn from a Gaussian centred on 0 with error $\delta_{\mathrm{PL}}$. 
Table 5. $P L$ relations of the form $M=\alpha+\beta \log P$.

\begin{tabular}{|c|c|c|c|c|c|}
\hline & $\alpha$ & $\beta$ & $N$ & Constraints & $\begin{array}{l}\text { LMC DM } \\
\text { (mag) }\end{array}$ \\
\hline \multicolumn{6}{|c|}{$\mathrm{FU}, 2.7<P(d)<35,|\mathrm{GOF}|<8, \epsilon_{i}<0.001, \delta_{\mathrm{PL}}$ applied. } \\
\hline 1 & $-1.919 \pm 0.119$ & $-2.386 \pm 0.138$ & 194 & $V$, GDR2, ZPoff $=0$ mas & \\
\hline 2 & $-1.875 \pm 0.118$ & $-2.305 \pm 0.136$ & 194 & $V$, GDR2, ZPoff $=-0.029$ mas & \\
\hline 3 & $-1.848 \pm 0.119$ & $-2.260 \pm 0.135$ & 194 & $V$, GDR2, ZPoff $=-0.046 \mathrm{mas}$ & \\
\hline 4 & $-2.912 \pm 0.058$ & $-3.154 \pm 0.070$ & 194 & $K, \mathrm{GDR} 2, \mathrm{ZPoff}=0 \mathrm{mas}$ & \\
\hline 5 & $-2.866 \pm 0.057$ & $-3.071 \pm 0.068$ & 194 & $K, \mathrm{GDR} 2, \mathrm{ZPoff}=-0.029 \mathrm{mas}$ & \\
\hline 6 & $-2.839 \pm 0.056$ & $-3.028 \pm 0.067$ & 194 & $K, \mathrm{GDR} 2, \mathrm{ZPoff}=-0.046 \mathrm{mas}$ & \\
\hline 7 & $-3.047 \pm 0.055$ & $-3.252 \pm 0.066$ & 194 & $W V K$, GDR2, ZPoff $=0$ mas & \\
\hline 8 & $-2.999 \pm 0.053$ & $-3.170 \pm 0.063$ & 194 & $W V K, \mathrm{GDR} 2, \mathrm{ZPoff}=-0.029 \mathrm{mas}$ & \\
\hline 9 & $-2.972 \pm 0.052$ & $-3.126 \pm 0.063$ & 194 & $W V K$, GDR2, ZPoff $=-0.046 \mathrm{mas}$ & \\
\hline 10 & $-1.917 \pm 0.118$ & $-2.351 \pm 0.137$ & 205 & $V$, GDR2+Ext, ZPoff $=0$ mas & \\
\hline 11 & $-2.908 \pm 0.057$ & $-3.109 \pm 0.068$ & 205 & $K$, GDR2+Ext, ZPoff $=0$ mas & \\
\hline 12 & $-3.041 \pm 0.053$ & $-3.207 \pm 0.063$ & 205 & $W V K$, GDR2+Ext, ZPoff $=0$ mas & \\
\hline 20 & $-1.728 \pm 0.029$ & -2.629 fixed & 194 & $V$, GDR 2, ZPoff $=0$ mas & \\
\hline 21 & $-1.619 \pm 0.029$ & -2.629 fixed & 194 & $V$, GDR2, ZPoff $=-0.029$ mas & \\
\hline 22 & $-1.557 \pm 0.029$ & -2.629 fixed & 194 & $V$, GDR2, ZPoff $=-0.046$ mas & \\
\hline 23 & $-1.690 \pm 0.029$ & -2.678 fixed & 194 & $V$, GDR2, ZPoff $=0$ mas & \\
\hline 24 & $-1.581 \pm 0.030$ & -2.678 fixed & 194 & $V$, GDR2, ZPoff $=-0.029 \mathrm{mas}$ & \\
\hline 25 & $-1.519 \pm 0.030$ & -2.678 fixed & 194 & $V$, GDR2, ZPoff $=-0.046 \mathrm{mas}$ & \\
\hline 26 & $-1.589 \pm 0.030$ & -2.810 fixed & 194 & $V$, GDR2, ZPoff $=0$ mas & $18.761 \pm 0.030$ \\
\hline 27 & $-1.480 \pm 0.030$ & -2.810 fixed & 194 & $V$, GDR2, ZPoff $=-0.029 \mathrm{mas}$ & 18.650 \\
\hline 28 & $-1.418 \pm 0.030$ & -2.810 fixed & 194 & $V$, GDR2, ZPoff $=-0.046 \mathrm{mas}$ & 18.590 \\
\hline 29 & $-1.321 \pm 0.030$ & -2.810 fixed & 194 & $V$, GDR2, ZPoff $=-0.074 \mathrm{mas}$ & 18.493 \\
\hline 30 & $-1.233 \pm 0.030$ & -2.810 fixed & 194 & $V$, GDR2, ZPoff $=-0.100 \mathrm{mas}$ & 18.405 \\
\hline 40 & $-2.879 \pm 0.014$ & -3.194 fixed & 194 & $K, \mathrm{GDR} 2, \mathrm{ZPoff}=0 \mathrm{mas}$ & $18.875 \pm 0.017$ \\
\hline 41 & $-2.769 \pm 0.014$ & -3.194 fixed & 194 & $K$, GDR2, ZPoff $=-0.029 \mathrm{mas}$ & 18.765 \\
\hline 42 & $-2.707 \pm 0.014$ & -3.194 fixed & 194 & $K, \mathrm{GDR} 2, \mathrm{ZPoff}=-0.046 \mathrm{mas}$ & 18.703 \\
\hline 43 & $-2.827 \pm 0.014$ & -3.260 fixed & 194 & $K, \mathrm{GDR} 2, \mathrm{ZPoff}=0 \mathrm{mas}$ & $18.880 \pm 0.014$ \\
\hline 44 & $-2.717 \pm 0.014$ & -3.260 fixed & 194 & $K$, GDR2, ZPoff $=-0.029 \mathrm{mas}$ & 18.770 \\
\hline 45 & $-2.655 \pm 0.014$ & -3.260 fixed & 194 & $K$, GDR2, ZPoff $=-0.046 \mathrm{mas}$ & 18.708 \\
\hline 46 & $-2.469 \pm 0.013$ & -3.260 fixed & 194 & $K, \mathrm{GDR} 2, \mathrm{ZPoff}=-0.100 \mathrm{mas}$ & 18.522 \\
\hline 47 & $-2.800 \pm 0.014$ & -3.295 fixed & 194 & $K, \mathrm{GDR} 2, \mathrm{ZPoff}=0 \mathrm{mas}$ & $18.870 \pm 0.022$ \\
\hline 48 & $-2.690 \pm 0.014$ & -3.295 fixed & 194 & $K, \mathrm{GDR} 2, \mathrm{ZPoff}=-0.029 \mathrm{mas}$ & 18.760 \\
\hline 49 & $-2.628 \pm 0.014$ & -3.295 fixed & 194 & $K, \mathrm{GDR} 2, \mathrm{ZPoff}=-0.046 \mathrm{mas}$ & 18.698 \\
\hline 50 & $-2.442 \pm 0.013$ & -3.295 fixed & 194 & $K, \mathrm{GDR} 2, \mathrm{ZPoff}=-0.100 \mathrm{mas}$ & 18.512 \\
\hline 51 & $-2.377 \pm 0.013$ & -3.295 fixed & 194 & $K$, GDR2, $\mathrm{ZPoff}=-0.120 \mathrm{mas}$ & 18.447 \\
\hline 52 & $-2.745 \pm 0.014$ & -3.365 fixed & 194 & $K, \mathrm{GDR} 2, \mathrm{ZPoff}=0 \mathrm{mas}$ & \\
\hline 52 & $-2.636 \pm 0.014$ & -3.365 fixed & 194 & $K, \mathrm{GDR} 2, \mathrm{ZPoff}=-0.029 \mathrm{mas}$ & \\
\hline 53 & $-2.574 \pm 0.014$ & -3.365 fixed & 194 & $K$, GDR2, ZPoff $=-0.046 \mathrm{mas}$ & \\
\hline 60 & $-2.997 \pm 0.013$ & -3.314 fixed & 194 & $W V K$, GDR2, ZPoff $=0$ mas & \\
\hline 61 & $-2.887 \pm 0.013$ & -3.314 fixed & 194 & $W V K$, GDR2, ZPoff $=-0.029 \mathrm{mas}$ & \\
\hline 62 & $-2.825 \pm 0.013$ & -3.314 fixed & 194 & $W V K$, GDR2, ZPoff $=-0.046 \mathrm{mas}$ & \\
\hline 63 & $-2.793 \pm 0.013$ & -3.314 fixed & 194 & $W V K$, GDR2, ZPoff $=-0.055 \mathrm{mas}$ & \\
\hline 64 & $-2.988 \pm 0.013$ & -3.325 fixed & 194 & $W V K$, GDR2, ZPoff $=0$ mas & $18.858 \pm 0.018$ \\
\hline 65 & $-2.878 \pm 0.013$ & -3.325 fixed & 194 & $W V K$, GDR2, ZPoff $=-0.029 \mathrm{mas}$ & 18.748 \\
\hline 66 & $-2.816 \pm 0.013$ & -3.325 fixed & 194 & $W V K$, GDR2, ZPoff $=-0.046 \mathrm{mas}$ & 18.696 \\
\hline 67 & $-2.784 \pm 0.012$ & -3.325 fixed & 194 & $W V K$, GDR2, ZPoff $=-0.055 \mathrm{mas}$ & 18.654 \\
\hline 68 & $-2.714 \pm 0.012$ & -3.325 fixed & 194 & $W V K$, GDR2, ZPoff $=-0.075 \mathrm{mas}$ & 18.584 \\
\hline 69 & $-2.630 \pm 0.012$ & -3.325 fixed & 194 & $W V K$, GDR2, ZPoff $=-0.100 \mathrm{mas}$ & 18.500 \\
\hline 70 & $-1.544 \pm 0.029$ & -2.629 fixed & 205 & $V$, GDR2+Ext, ZPoff $=-0.046 \mathrm{mas}$ & \\
\hline 71 & $-1.506 \pm 0.030$ & -2.678 fixed & 205 & $V$, GDR2+Ext, ZPoff $=-0.046 \mathrm{mas}$ & \\
\hline 72 & $-1.404 \pm 0.030$ & -2.810 fixed & 205 & $V$, GDR2+Ext, ZPoff $=-0.046$ mas & $18.576 \pm 0.030$ \\
\hline 73 & $-2.684 \pm 0.013$ & -3.194 fixed & 205 & $K$, GDR2+Ext, ZPoff $=-0.046$ mas & \\
\hline 74 & $-2.632 \pm 0.013$ & -3.260 fixed & 205 & $K$, GDR2+Ext, ZPoff $=-0.046 \mathrm{mas}$ & $18.685 \pm 0.013$ \\
\hline 75 & $-2.604 \pm 0.013$ & -3.295 fixed & 205 & $K, \mathrm{GDR} 2+\mathrm{Ext}, \mathrm{ZPoff}=-0.046 \mathrm{mas}$ & \\
\hline 76 & $-2.550 \pm 0.013$ & -3.365 fixed & 205 & $K$, GDR2+Ext, ZPoff $=-0.046$ mas & \\
\hline 77 & $-2.800 \pm 0.012$ & -3.314 fixed & 205 & $W V K$, GDR2+Ext, ZPoff $=-0.046 \mathrm{mas}$ & \\
\hline 78 & $-2.792 \pm 0.012$ & -3.325 fixed & 205 & $W V K$, GDR2+Ext, ZPoff $=-0.046 \mathrm{mas}$ & $18.662 \pm 0.018$ \\
\hline
\end{tabular}

Notes. $N$ is the number of stars in the solution. 
M. A. T. Groenewegen et al.: The Cepheid period-luminosity-metallicity relation based on Gaia DR2 data

Table 6. $P L(Z)$ relations in the literature of the form $M=\alpha+\beta \log P+\gamma[\mathrm{Fe} / \mathrm{H}]$ for different galaxies.

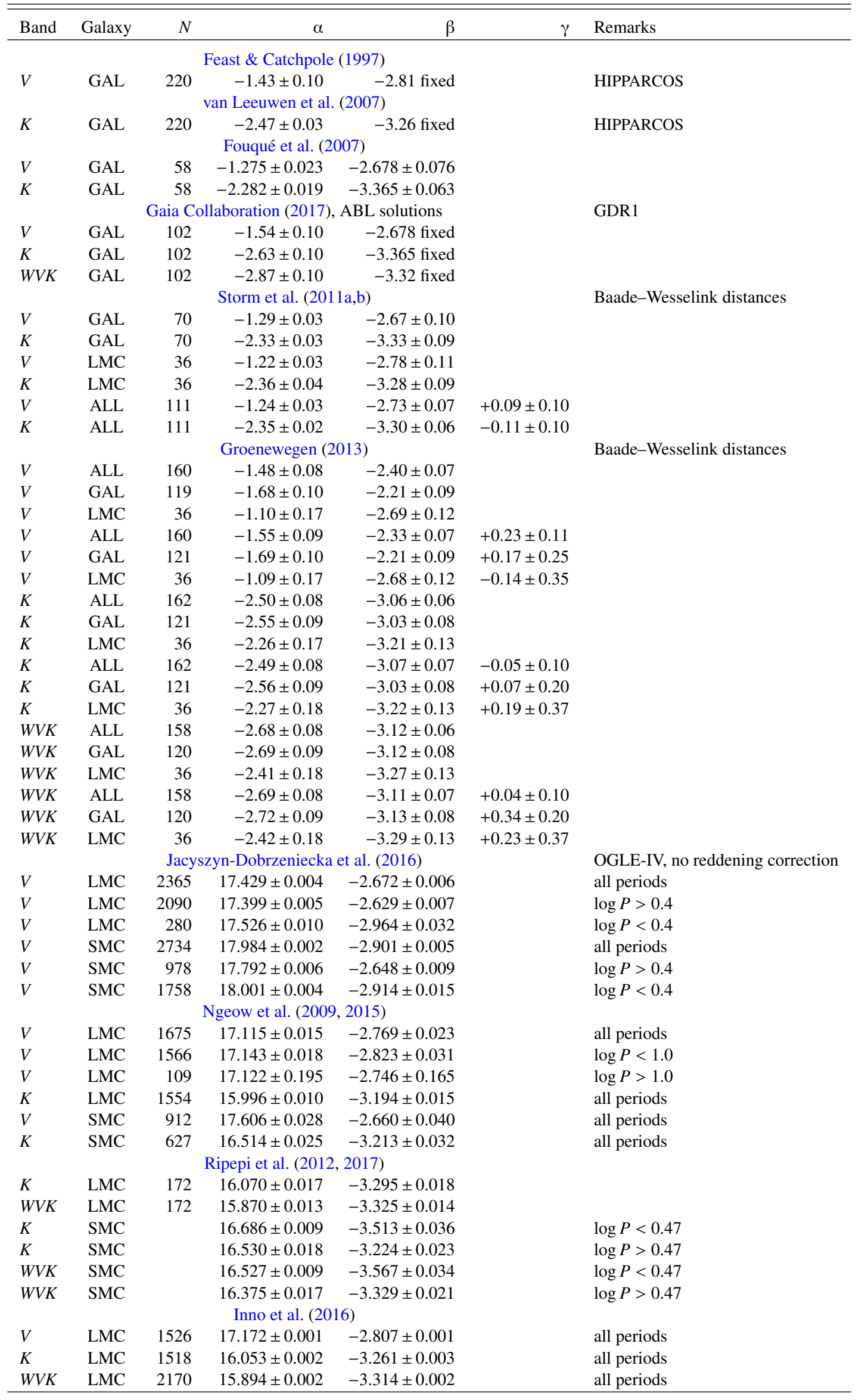


The generated data ( $\pi, \sigma_{\pi}$, magnitude, period, colour, iron abundance) are fed to the minimization routine that returns the parameters $(\alpha, \beta, \gamma)$ with internal error bars. This is repeated $N$ times (typically $N=1001)$. The best estimate of the parameters is taken as the median of its distribution with the $1 \sigma$ error bar derived from the $2.7 \%$ and $93.7 \%$ percentiles $( \pm 2 \sigma)$. This results in realistic error bars that takes into account all likely variations in the data.

Using the best fit parameters $(\alpha, \beta, \gamma$ for each simulation) the photometric parallax is calculated using Eqs. (1) and (2) and compared to the observed parallax. This gives a standard deviation per star and when these values are ordered over all simulations, stars that are systematic outliers can be identified. An rms deviation of the fit to the data is also calculated.

\section{Simulations}

To test the methodology and the sensitivity to the parameters, simulations were carried out. Parallax and error in the parallax are generated based on the period, reddening, magnitudes of the stars in the sample, and a period-luminosity relation. These observed parameters are then fed to the code and analysed as described above. The results are listed in Table 4 . The sample size was chosen to represent the number of FU and FO pulsators in the sample. Solutions 1-4 are idealised: the simulated parallax is exactly $1 /$ distance, and the error in the parallax is based on a fraction of the distance. The input slope and zero point are retrieved almost exactly. In all other simulations the simulated parallax is based on the exact parallax plus a Gaussian distributed error. In solutions $5-8$ this is still based on a hypothetical fractional error in the distance. In all other simulations the error in the parallax is based on the observed distribution among the stars with a GOF $<8$. The distribution is not a strong function of $G$-band (or $V$-band) magnitude in the present sample, and $\sigma_{\pi}$ is represented by a mean of 0.040 and a Gaussian dispersion of 0.013 with a minimum value of 0.020 mas. Solution 9 is the standard case which returns the input slope and zero point with a slight offset, but still within the error bars. Selecting on parallax error does not influence this (solutions 12 and 13. We note that in the present sample, very few or no negative parallaxes are predicted).

The last part of the simulations investigates the influence of a zero-point offset in the parallax. The all-sky average value derived from QSOs (-0.029 mas; Lindegren et al. 2018) and for a sample of 50 CCs ( -0.046 mas; Riess et al. 2018b) are used to illustrate this.

The impact is significant on both zero point and slope of the $P L$ relation when not considered in the analysis of the data (solutions 14 and 15). The true solution can be partially recovered when the parallax zero-point offset is partially taken into account in the analysis (solutions 16 and 17). Making a strong selection on the relative parallax error or parallax may also recover an unrecognised parallax zero-point offset, but at the expense of a larger error bar in the zero point and slope of the $P L$ relation (solutions 18-21 and 22-24).

The last two entries are for a realistic sample size (see next section), also including an intrinsic width of the instability strip (IS). In both cases this leads to larger error bars in the derived parameters.

\section{Results}

\subsection{PL relation}

Two sources of parallax data are considered. The first is exclusively based on GDR2 data. However, many bright stars have

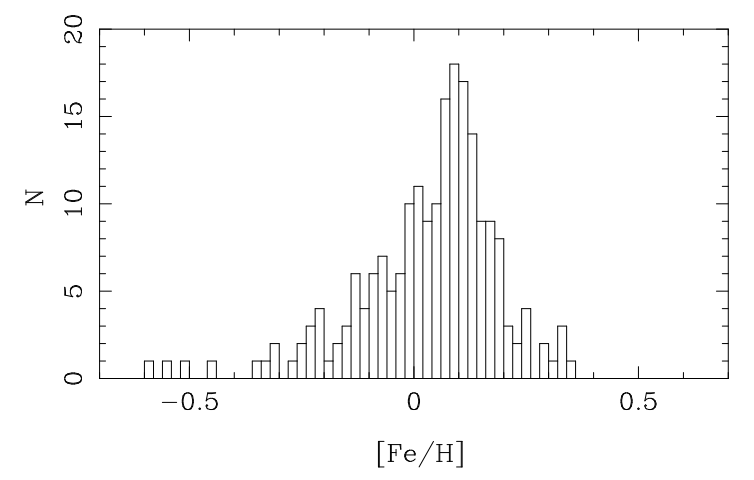

Fig. 4. Distribution of the $[\mathrm{Fe} / \mathrm{H}]$ abundance in the reduced sample of 205 objects.

a very poor astrometric solution and have to be discarded (see Table 3 and the discussion below). In the second sample the poorest GDR2 parallax data (with GOF $>8$ ) is replaced by external data when available. These are the 11 stars with an entry in the last column of Table 3 (as having a non-HIPPARCOS based accurate parallax) and 9 stars where the weighted mean of the available HIPPARCOS parallaxes was used ${ }^{3}$.

For the moment we do not consider the metallicity and concentrate on the classical $P L$ relation in the $V, K$, and $W V K$ bands, the Wesenheit reddening-free index defined as $W V K=K-$ $0.13(V-K)$ (Ripepi et al. 2012), based on the reddening law of Cardelli et al. (1989). A large number of models was run to investigate the influence of FU versus FO pulsators, period cuts, selection on GOF, the parallax zero-point offset, the intrinsic width of the IS, and to identify systematic outliers. For completeness the model results are reported and briefly discussed in Appendix A. The conclusions are to conservatively use only FU mode pulsators (the FO mode pulsators seem to show a significantly different slope), consider stars between 2.7 and 35 days (mainly for the comparison to SMC and LMC results), and select stars with $|\mathrm{GOF}|<8$ and $\epsilon_{i}<0.001$. The stars U Vul, V Vel, V636 Cas, V526 Aql, QQ Per, and HQ Car are also excluded. The last two are likely T2C. The standard values for $\delta_{\mathrm{PL}}$ of $0.20,0.066$, and 0.049 mag in the $V, K$, and $W V K$ bands are adopted below (Inno et al. 2016).

Table 5 lists the results for this reduced sample of stars. Solutions 1-9 using only GDR2 parallaxes (marked "GDR2" in the table), solutions 10-12 using external parallaxes when available for the poorest GDR2 data (marked "GDR2+Ext". Any parallax zero-point offset is only applied to the GDR2 objects). In these first solutions zero point and slope of the $P L$ relations are both fitted. For a fixed parallax zero-point offset adding the external parallax data does not have a very big effect (less then $1 \sigma$; solutions $1,4,7$ versus $10,11,12$ ).

The effect of a parallax zero-point offset is larger, and systematic. Increasing the Gaia parallax (making the offset more negative in the convention used by Lindegren et al. 2018) makes the slope less steep and the zero point fainter in all three filters considered.

The slope of the $P L$ relation derived in the $V, K$, and $W V K$ filters is different than that in the LMC. Table 6 compiles recent determinations of the $P L(Z)$ relation in these filters in the

\footnotetext{
3 Specifically: AH Vel $1.454 \pm 0.174$ mas, BG Cru 2.282 \pm 0.209 mas, DT Cyg 2.060 \pm 0.233 mas, $\eta$ Aql $3.150 \pm 0.579$ mas, MY Pup 1.097 \pm 0.143 mas, SU Cas $2.549 \pm 0.229$ mas, R Cru $2.060 \pm 0.480$ mas, and S Mus $2.120 \pm 0.350$ mas. Only HIPPARCOS parallax solutions with a GOF $<3.5$ and relative parallax error below $25 \%$ were considered in the averaging.
} 
Table 7. $P L$ relations of the form $M=\alpha+\beta \log P+\gamma[\mathrm{Fe} / \mathrm{H}]$.

\begin{tabular}{|c|c|c|c|c|c|c|}
\hline & $\alpha$ & $\beta$ & $\gamma$ & $N$ & Constraints & $\begin{array}{l}\text { LMC DM } \\
\text { (mag) }\end{array}$ \\
\hline \multicolumn{7}{|c|}{ FU, $2.7<P(d)<35,|\mathrm{GOF}|<8, \epsilon_{i}<0.001, \delta_{\mathrm{PL}}$ applied } \\
\hline 101 & $-1.929 \pm 0.120$ & $-2.407 \pm 0.143$ & $0.326 \pm 0.257$ & 194 & $V$, GDR2, ZPoff $=0$ mas & \\
\hline 102 & $-1.879 \pm 0.121$ & $-2.312 \pm 0.142$ & $0.117 \pm 0.254$ & 194 & $V$, GDR2, ZPoff $=-0.029$ mas & \\
\hline 103 & $-1.846 \pm 0.120$ & $-2.259 \pm 0.141$ & $0.001 \pm 0.258$ & 194 & $V$, GDR2, ZPoff $=-0.046$ mas & \\
\hline 104 & $-2.917 \pm 0.060$ & $-3.166 \pm 0.074$ & $0.237 \pm 0.171$ & 194 & $K$, GDR2, ZPoff $=0$ mas & \\
\hline 105 & $-2.865 \pm 0.057$ & $-3.072 \pm 0.072$ & $0.027 \pm 0.164$ & 194 & $K$, GDR2, ZPoff $=-0.029 \mathrm{mas}$ & \\
\hline 106 & $-2.835 \pm 0.057$ & $-3.020 \pm 0.071$ & $-0.090 \pm 0.159$ & 194 & $K$, GDR2, ZPoff $=-0.046 \mathrm{mas}$ & \\
\hline 107 & $-3.051 \pm 0.055$ & $-3.266 \pm 0.071$ & $0.223 \pm 0.167$ & 194 & $W V K$, GDR2, ZPoff $=0$ mas & \\
\hline 108 & $-2.999 \pm 0.053$ & $-3.171 \pm 0.067$ & $0.015 \pm 0.161$ & 194 & $W V K$, GDR2, ZPoff $=-0.029 \mathrm{mas}$ & \\
\hline 109 & $-2.968 \pm 0.052$ & $-3.120 \pm 0.066$ & $-0.102 \pm 0.156$ & 194 & $W V K$, GDR2, ZPoff $=-0.046 \mathrm{mas}$ & \\
\hline 110 & $-1.916 \pm 0.119$ & $-2.359 \pm 0.136$ & $0.106 \pm 0.239$ & 205 & $V$, GDR2+Ext, ZPoff $=0$ mas & \\
\hline 111 & $-1.834 \pm 0.118$ & $-2.250 \pm 0.136$ & $-0.064 \pm 0.236$ & 205 & $V$, GDR2+Ext, ZPoff $=-0.046 \mathrm{mas}$ & \\
\hline 112 & $-2.907 \pm 0.058$ & $-3.109 \pm 0.069$ & $-0.003 \pm 0.163$ & 205 & $K, \mathrm{GDR} 2+\mathrm{Ext}, \mathrm{ZPoff}=0 \mathrm{mas}$ & \\
\hline 113 & $-2.824 \pm 0.057$ & $-2.999 \pm 0.067$ & $-0.189 \pm 0.139$ & 205 & $K$, GDR2+Ext, ZPoff $=-0.046 \mathrm{mas}$ & \\
\hline 114 & $-3.041 \pm 0.053$ & $-3.206 \pm 0.065$ & $-0.013 \pm 0.160$ & 205 & $W V K$, GDR2+Ext, ZPoff $=0$ mas & \\
\hline 115 & $-2.976 \pm 0.052$ & $-3.096 \pm 0.062$ & $-0.204 \pm 0.140$ & 205 & $W V K$, GDR $2+$ Ext, ZPoff $=-0.046$ mas & \\
\hline 120 & $-1.617 \pm 0.033$ & -2.810 fixed & $0.388 \pm 0.271$ & 194 & $V$, GDR2, ZPoff $=0$ mas & $18.925 \pm 0.102$ \\
\hline 121 & $-1.494 \pm 0.033$ & -2.810 fixed & $0.197 \pm 0.278$ & 194 & $V$, GDR2, ZPoff $=-0.029 \mathrm{mas}$ & $18.735 \pm 0.103$ \\
\hline 122 & $-1.424 \pm 0.034$ & -2.810 fixed & $0.095 \pm 0.284$ & 194 & $V$, GDR2, ZPoff $=-0.046$ mas & $18.629 \pm 0.105$ \\
\hline 125 & $-2.846 \pm 0.019$ & -3.260 fixed & $0.252 \pm 0.166$ & 194 & $K, \mathrm{GDR} 2, \mathrm{ZPoff}=0 \mathrm{mas}$ & $18.987 \pm 0.062$ \\
\hline 126 & $-2.721 \pm 0.017$ & -3.260 fixed & $0.054 \pm 0.162$ & 194 & $K$, GDR2, ZPoff $=-0.029 \mathrm{mas}$ & $18.792 \pm 0.059$ \\
\hline 127 & $-2.651 \pm 0.017$ & -3.260 fixed & $-0.053 \pm 0.161$ & 194 & $K$, GDR2, ZPoff $=-0.046 \mathrm{mas}$ & $18.685 \pm 0.059$ \\
\hline 130 & $-3.006 \pm 0.018$ & -3.325 fixed & $0.231 \pm 0.166$ & 194 & $W V K$, GDR2, ZPoff $=0$ mas & $18.957 \pm 0.063$ \\
\hline 131 & $-2.881 \pm 0.016$ & -3.325 fixed & $0.038 \pm 0.156$ & 194 & $W V K$, GDR2, ZPoff $=-0.029$ mas & $18.764 \pm 0.058$ \\
\hline 132 & $-2.811 \pm 0.016$ & -3.325 fixed & $-0.070 \pm 0.157$ & 194 & $W V K$, GDR2, ZPoff $=-0.046 \mathrm{mas}$ & $18.656 \pm 0.059$ \\
\hline 140 & $-1.566 \pm 0.034$ & -2.810 fixed & $0.136 \pm 0.250$ & 205 & $V$, GDR2+Ext, ZPoff $=0$ mas & $18.786 \pm 0.094$ \\
\hline 141 & $-1.460 \pm 0.034$ & -2.810 fixed & $0.020 \pm 0.254$ & 205 & $V$, GDR2+Ext, ZPoff $=-0.029$ mas & $18.639 \pm 0.095$ \\
\hline 142 & $-1.400 \pm 0.033$ & -2.810 fixed & $-0.041 \pm 0.260$ & 205 & $V$, GDR2+Ext, ZPoff $=-0.046 \mathrm{mas}$ & $18.558 \pm 0.097$ \\
\hline 145 & $-2.789 \pm 0.017$ & -3.260 fixed & $0.013 \pm 0.163$ & 205 & $K, \mathrm{GDR} 2+\mathrm{Ext}, \mathrm{ZPoff}=0$ mas & $18.847 \pm 0.060$ \\
\hline 146 & $-2.681 \pm 0.016$ & -3.260 fixed & $-0.106 \pm 0.148$ & 205 & $K$, GDR2+Ext, ZPoff $=-0.029 \mathrm{mas}$ & $18.697 \pm 0.055$ \\
\hline 147 & $-2.620 \pm 0.016$ & -3.260 fixed & $-0.168 \pm 0.146$ & 205 & $K$, GDR2+Ext, ZPoff $=-0.046 \mathrm{mas}$ & $18.614 \pm 0.054$ \\
\hline 148 & $-2.599 \pm 0.016$ & -3.260 fixed & $-0.193 \pm 0.143$ & 205 & $K$, GDR2+Ext, ZPoff $=-0.052 \mathrm{mas}$ & $18.584 \pm 0.053$ \\
\hline 150 & $-2.948 \pm 0.017$ & -3.325 fixed & $-0.002 \pm 0.156$ & 205 & $W V K$, GDR $2+$ Ext, $\mathrm{ZPoff}=0 \mathrm{mas}$ & $18.817 \pm 0.058$ \\
\hline 151 & $-2.840 \pm 0.015$ & -3.325 fixed & $-0.125 \pm 0.146$ & 205 & $W V K$, GDR2+Ext, ZPoff $=-0.029$ mas & $18.666 \pm 0.055$ \\
\hline 152 & $-2.779 \pm 0.015$ & -3.325 fixed & $-0.188 \pm 0.142$ & 205 & $W V K$, GDR $2+$ Ext, ZPoff $=-0.046$ mas & $18.583 \pm 0.058$ \\
\hline 153 & $-2.758 \pm 0.015$ & -3.325 fixed & $-0.211 \pm 0.141$ & 205 & $W V K$, GDR2+Ext, ZPoff $=-0.052$ mas & $18.554 \pm 0.054$ \\
\hline 154 & $-2.713 \pm 0.015$ & -3.325 fixed & $-0.260 \pm 0.140$ & 205 & $W V K$, GDR2+Ext, ZPoff $=-0.065$ mas & $18.492 \pm 0.054$ \\
\hline
\end{tabular}

Notes. $N$ is the number of stars in the solution.

SMC, LMC, and the Milky Way. They include the zero point and slope derived for Galactic Cepheids using HIPPARCOS data (Feast \& Catchpole 1997; van Leeuwen et al. 2007), the results by Fouqué et al. (2007) that have been used in the analysis of GDR1 data in Gaia Collaboration (2017). Listed next are the results by Storm et al. (2011a,b) and Groenewegen (2013) from Baade-Wesselink distances to SMC, LMC, and Galactic objects. The solutions for the Galactic and LMC objects are listed, and the solutions for all objects, which then includes a few CCs in the SMC as well. The last sets of entries are based on OGLE data in the $V$ band, sometimes combined with sets of NIR photometry. We note that there is no single slope (and zero point) for the LMC (and SMC) Cepheids. The values depend on the period cut used, but also on more seemingly subtle issues. Inno et al. (2016) discuss the differences with Jacyszyn-Dobrzeniecka et al. (2016) which both use the OGLE-IV catalogue of CCs. In the $I$ band the papers find different slopes $(-3.327 \pm 0.001$ versus $-3.313 \pm 0.006)$ and Inno et al. (2016) trace this back to a difference in the sigmaclipping procedure (removing $3 \sigma$ versus $6 \sigma$ outliers).
In the $V$ band the difference in slope between the LMC $(\sim-2.65$ to -2.8$)$ and the GDR2 results $(\sim-2.35$ to -2.38$)$ is at the $3-4 \sigma$ level, and larger when applying a parallax zero-point offset. On the other hand, of the three bands considered the $V$ band is the one where the evidence for non-linearity is strongest and where the slope of SMC Cepheids is known to be inconsistent with that of LMC Cepheids (Ngeow et al. 2009, 2015). In the $K$ band the difference in slope between the LMC $(\sim-3.20$ to -3.35$)$ and the GDR2 results ( -3.10 to -3.15$)$ is at the $1-2 \sigma$ level, but becomes significant when applying a parallax zero-point offset. In the $W V K$ band the same tendency is seen, the difference in slope between the LMC and the GDR2 results becomes significant when applying a parallax zero-point offset.

In solution 20 and in Table 5 the slope of the $P L$ relation has been fixed to different values found for LMC Cepheids. This has been done for both sources of parallax data, and for different parallax zero-point offsets. For a fixed slope, the difference between the zero points gives the distance modulus (DM) to the LMC, and this is listed in the last column for selected models. The 
Table 8. $P L$ relations of the form $M=\alpha+\beta \log P+\gamma[\mathrm{Fe} / \mathrm{H}]$ and variation in the parameters.

\begin{tabular}{|c|c|c|c|c|c|c|}
\hline & $\alpha$ & $\beta$ & $\gamma$ & $N$ & Constraints & $\begin{array}{l}\text { LMC DM } \\
\text { (mag) }\end{array}$ \\
\hline \multicolumn{7}{|c|}{ Standard: FU, $2.7<P(d)<35,|\mathrm{GOF}|<8, \epsilon_{i}<0.001, \delta_{\mathrm{PL}}$ applied, GDR2+Ext set, ZPoff $=-0.049$ mas } \\
\hline 160 & $-1.390 \pm 0.033$ & -2.810 fixed & $-0.051 \pm 0.260$ & 205 & $V$, standard & $18.544 \pm 0.097$ \\
\hline 161 & $-1.470 \pm 0.060$ & -2.810 fixed & $-0.042 \pm 0.453$ & 205 & $V, \delta_{\mathrm{PL}} \cdot 2=0.40 \mathrm{mag}$ & $18.627 \pm 0.170$ \\
\hline 162 & $-1.372 \pm 0.035$ & -2.810 fixed & $-0.149 \pm 0.280$ & 205 & $V, \sigma_{\pi} \cdot 1.5$ for GDR2 & $18.492 \pm 0.104$ \\
\hline 163 & $-1.442 \pm 0.032$ & -2.810 fixed & $-0.084 \pm 0.248$ & 205 & $V, E(B-V) \cdot 1.05$ & $18.585 \pm 0.093$ \\
\hline 164 & $-1.394 \pm 0.039$ & -2.810 fixed & $-0.005 \pm 0.264$ & 205 & $V$, other pick of Iron abundance & $18.564 \pm 0.100$ \\
\hline 165 & $-1.388 \pm 0.033$ & -2.810 fixed & $-0.060 \pm 0.268$ & 205 & $V, \sigma_{[\mathrm{Fe} / \mathrm{H}]}=0.04 \mathrm{dex}$ & $18.539 \pm 0.099$ \\
\hline 166 & $-1.405 \pm 0.053$ & -2.810 fixed & $-0.230 \pm 0.443$ & 28 & $V, \sigma_{\pi} / \pi<0.05$ & $18.497 \pm 0.164$ \\
\hline 167 & $-1.389 \pm 0.033$ & -2.810 fixed & $+0.010 \pm 0.262$ & 208 & $V, P>2.7 \mathrm{~d}$ & $18.565 \pm 0.097$ \\
\hline 168 & $-1.385 \pm 0.033$ & -2.810 fixed & $-0.047 \pm 0.243$ & 217 & $V, P>2.0 \mathrm{~d}$ & $18.541 \pm 0.091$ \\
\hline 170 & $-2.610 \pm 0.016$ & -3.260 fixed & $-0.182 \pm 0.145$ & 205 & $K$, standard & $18.599 \pm 0.054$ \\
\hline 171 & $-2.618 \pm 0.023$ & -3.260 fixed & $-0.183 \pm 0.177$ & 205 & $K, \delta_{\mathrm{PL}} \cdot 2=0.13 \mathrm{mag}$ & $18.607 \pm 0.067$ \\
\hline 172 & $-2.584 \pm 0.019$ & -3.260 fixed & $-0.279 \pm 0.164$ & 205 & $K, \sigma_{\pi} \cdot 1.5$ for GDR2 & $18.539 \pm 0.062$ \\
\hline 173 & $-2.614 \pm 0.016$ & -3.260 fixed & $-0.185 \pm 0.146$ & 205 & $K, E(B-V) \cdot 1.05$ & $18.602 \pm 0.054$ \\
\hline 174 & $-2.591 \pm 0.016$ & -3.260 fixed & $-0.171 \pm 0.143$ & 205 & $K, A_{K} / A_{V}=0.10$ & $18.584 \pm 0.053$ \\
\hline 175 & $-2.611 \pm 0.019$ & -3.260 fixed & $-0.113 \pm 0.157$ & 205 & $K$, other pick of Iron abundance & $18.624 \pm 0.058$ \\
\hline 176 & $-2.604 \pm 0.016$ & -3.260 fixed & $-0.259 \pm 0.140$ & 205 & $K, \sigma_{[\mathrm{Fe} / \mathrm{H}]}=$ & $18.566 \pm 0.053$ \\
\hline 177 & $-2.602 \pm 0.025$ & -3.260 fixed & $-0.267 \pm 0.260$ & 28 & $K, \sigma_{\pi} / \pi<0.05$ & $18.562 \pm 0.095$ \\
\hline 178 & $-2.609 \pm 0.017$ & -3.260 fixed & $-0.141 \pm 0.151$ & 208 & $K, P>2.7 \mathrm{~d}$ & $18.612 \pm 0.056$ \\
\hline 179 & $-2.605 \pm 0.016$ & -3.260 fixed & $-0.207 \pm 0.146$ & 217 & $K, P>2.0 \mathrm{~d}$ & $18.586 \pm 0.055$ \\
\hline 180 & $-2.769 \pm 0.015$ & -3.325 fixed & $-0.200 \pm 0.141$ & 205 & $W V K$, standard & $18.569 \pm 0.054$ \\
\hline 181 & $-2.773 \pm 0.019$ & -3.325 fixed & $-0.197 \pm 0.157$ & 205 & $W V K, \delta_{\mathrm{PL}} \cdot 2=0.09 \mathrm{mag}$ & $18.574 \pm 0.060$ \\
\hline 182 & $-2.741 \pm 0.018$ & -3.325 fixed & $-0.297 \pm 0.166$ & 205 & $W V K, \sigma_{\pi} \cdot 1.5$ for GDR2 & $18.507 \pm 0.064$ \\
\hline 183 & $-2.769 \pm 0.015$ & -3.325 fixed & $-0.200 \pm 0.141$ & 205 & $W V K, E(B-V) \cdot 1.05$ & $18.569 \pm 0.054$ \\
\hline 184 & $-2.748 \pm 0.015$ & -3.325 fixed & $-0.186 \pm 0.141$ & 205 & $W V K, A_{K} / A_{V}=0.10$ & $18.553 \pm 0.054$ \\
\hline 185 & $-2.770 \pm 0.019$ & -3.325 fixed & $-0.126 \pm 0.155$ & 205 & $W V K$, other pick of Iron abundance & $18.596 \pm 0.059$ \\
\hline 186 & $-2.762 \pm 0.015$ & -3.325 fixed & $-0.285 \pm 0.133$ & 205 & $W V K, \sigma_{[\mathrm{Fe} / \mathrm{H}]}=0.04 \mathrm{dex}$ & $18.532 \pm 0.053$ \\
\hline 187 & $-2.768 \pm 0.015$ & -3.325 fixed & $-0.199 \pm 0.143$ & 205 & $W V K, \sigma_{V} \cdot 2, \sigma_{K} \cdot 2$ & $18.568 \pm 0.055$ \\
\hline 188 & $-2.784 \pm 0.015$ & -3.325 fixed & $-0.191 \pm 0.145$ & 205 & $W V K$, No NIR transformation & $18.587 \pm 0.055$ \\
\hline 189 & $-2.756 \pm 0.024$ & -3.325 fixed & $-0.273 \pm 0.247$ & 28 & $W V K, \sigma_{\pi} / \pi<0.05$ & $18.530 \pm 0.092$ \\
\hline 190 & $-2.768 \pm 0.016$ & -3.325 fixed & $-0.158 \pm 0.147$ & 208 & $W V K, P>2.7 \mathrm{~d}$ & $18.583 \pm 0.056$ \\
\hline 191 & $-2.763 \pm 0.015$ & -3.325 fixed & $-0.224 \pm 0.140$ & 217 & $W V K, P>2.0 \mathrm{~d}$ & $18.555 \pm 0.054$ \\
\hline 200 & $-1.840 \pm 0.118$ & $-2.243 \pm 0.137$ & 0.0 fixed & 205 & $V$, standard & \\
\hline 201 & $-2.827 \pm 0.055$ & $-3.000 \pm 0.065$ & 0.0 fixed & 205 & $K$, standard & \\
\hline 202 & $-2.961 \pm 0.051$ & $-3.098 \pm 0.060$ & 0.0 fixed & 205 & $W V K$, standard & \\
\hline
\end{tabular}

Notes. $N$ is the number of stars in the solution.

quoted error considers the error in both the Galactic and LMC zero points. The DM depend strongly on the adopted parallax zero-point offset. For an offset of -0.046 mas the DM is essentially 18.70 mag based on the $K$ and $W V K$ band (it is $0.1 \mathrm{mag}$ shorter but with a larger error bar in $V$ ). Adding the external parallax data makes these distances shorter by about $0.02 \mathrm{mag}$. The current analysis suggests that a DM of $18.493 \pm 0.048$ mag (based on the eclipsing binaries; Pietrzyński et al. 2013) or the recommended value of $18.49 \pm 0.09 \mathrm{mag}$ (based on several independent distance indicators; de Grijs et al. 2014) requires a significantly larger offset (in absolute sense) of order -0.1 mas. This is comparable to the $-0.118 \pm 0.003$ listed in Arenou et al. (2018) for the difference between GDR2 and HIPPARCOS parallaxes, but larger (in absolute sense) than given by any of the other comparisons listed in their Table 1.

\subsection{PLZ relation}

In this section the effect of metallicity is considered. Figure 4 shows the histogram over the $[\mathrm{Fe} / \mathrm{H}]$ abundance of the reduced sample. The abundance ranges from -0.59 to +0.34 with an average of +0.03 and a median of +0.06 . The mean abundance of $\mathrm{CCs}$ in the LMC with individually determined accurate metallicities (22 stars from Luck et al. 1998; Romaniello et al. 2008; Lemasle et al. 2017) is $-0.35 \pm 0.05$.

Table 7 contains the results when fitting the PLZ relation. The content is similar to Table 5; solutions have been derived by either fitting or fixing the slope of the period dependence, for the two sources of parallax data and for various parallax zero-point offsets. For the solutions with fixed slope the DM to the LMC is listed in the last column, using the mean abundance and including the error on the mean abundance for the LMC Cepheids in the error budget.

The parallax zero-point offset again has an important influence on the results. The zero point and slope of the period dependence change only marginally when also fitting for the metallicity dependence, and the metallicity dependence itself is absent to marginal $(\lesssim 1.5 \sigma)$, but there is a degeneracy of the parameters. Adding the external parallax data makes the metallicity effect stronger. 
Solutions 120 and higher are calculated for a fixed period dependence to be able to calculate the DM to the LMC. The result is a stronger dependence on metallicity (in an absolute sense) with, depending on the parallax zero-point offset and the filter, a significance that ranges from non-existing to an $\sim 2 \sigma$ effect. For a given parallax zero-point offset the LMC DM is found to be smaller by $\sim 0.03-0.08$ mag than when excluding the metallicity term.

\section{Discussion and summary}

From an initial sample of 452 Galactic Cepheids with accurate $[\mathrm{Fe} / \mathrm{H}]$ abundances, period-luminosity and period-luminositymetallicity relations have been derived based on parallax data from Gaia DR2, supplemented with accurate non-Gaia parallax data when available, for a final sample of about $200 \mathrm{FU}$ mode Cepheids with good astrometric solutions.

The influence of a parallax zero-point offset on the derived $P L(Z)$ relation is large, which means that the current GDR2 results do not allow us to improve on the existing calibration of the relation or on the distance to the LMC. The zero point, the slope of the period dependence, and any metallicity dependence of the $P L(Z)$ relations are correlated with any assumed parallax zero-point offset.

Based on a comparison for nine CCs with the best nonGaia parallaxes (mostly from HST data) a parallax zeropoint offset of $-0.049 \pm 0.018$ mas, consistent with other values that appeared in the literature after the release of GDR2, is derived from RGB stars using Kepler and APOGEE data (about -0.053 mas; Zinn et al. 2018), eclipsing binaries $(-0.082 \pm 0.033$ mas; Stassun \& Torres 2018), a sample of 50 CCs (-0.046 \pm 0.013 mas; Riess et al. 2018b), and RR Lyrae stars ( - 0.056 mas; Muraveva et al. 2018).

For a parallax zero-point offset of -0.049 mas a final list of calculations, investigating the influence of some other parameters is given Table 8 . The slope of the period dependence has been fixed (solutions 160-190), and the resulting DM to the LMC is listed in the last column. Variations in the assumed dispersion in the photometry, reddening, or period cuts have a relatively small impact on the results (especially in $K$ and $W V K$ ). These models address some concerns that might arise when combining many sources of photometry, reddening, and iron abundances from the literature. Increasing the error in the mean $V$ and $K$ magnitude, or not applying a transformation of the different NIR magnitude systems at all has very little impact.

The most noticeable effect is when the parallax errors in GDR2 are underestimated as this changes the weight of the GDR2 sample with respect to the stars with a non-Gaia parallax. Lindegren et al. (2018) in their Appendix A hint at the fact that the errors in the five astrometric parameters may still be underestimated even after correcting for the DOF bug. This issue will likely be resolved in future releases. The next most important effect is the iron abundance. A smaller error in its determination would help, but equally important is a homogeneous metallicity scale (see Proxauf et al. 2018 for new efforts in this direction).

Solutions 200-202 give the current best estimate of the $P L$ relation (for the assumed parallax zero-point offset), without a metallicity dependence as the current data and analysis does not allow us to prove or disprove a dependence. Figure 5 shows these relations in the three bands considered. No additional sigmaclipping in magnitude space has been applied (as is common in deriving $P L$ relations in the LMC). Only stars that were systematic outliers in $\sigma$-space have been removed, as explained in
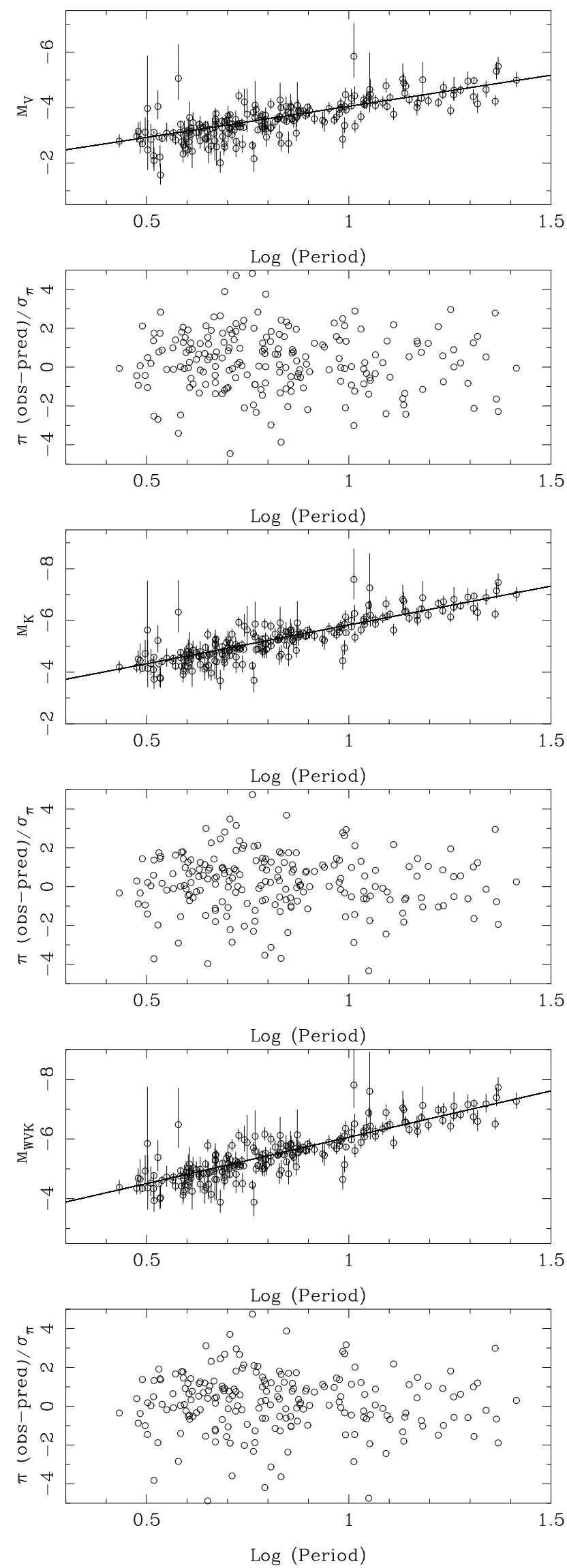

Fig. 5. $P L$ relations in the $V, K$, and $W V K$ bands (solutions 200-202 from Table 8). For each filter the second panel gives the deviation between observed and predicted parallax (limited to $\pm 5 \sigma$ ). 
Sect. 6. As noted before, the slopes of the galactic $P L$ relation are shallower than those derived in the LMC by $\sim 3 \sigma$ (for the assumed parallax zero-point offset). If the slope is fixed to values that have been derived for LMC Cepheids, the derived parallax zero-point offset suggests a LMC DM that is larger than commonly adopted. Conversely, a LMC DM of around 18.50 requires a zero-point offset closer to -0.1 mas.

The results in this paper show that the parallax zero-point offset should be known to a level of $\lesssim 3 \mu$ as to have a $\lesssim 0.01$ mag effect on zero point of the $P L$ relation and the DM to the LMC. It will remain important to have accurate non-Gaia based parallaxes as a control sample, like the ongoing programme using HST (Riess et al. 2014, 2018a; Casertano et al. 2016).

Acknowledgements. This work has made use of data from the European Space Agency (ESA) mission Gaia (http://www.cosmos.esa.int/gaia), processed by the Gaia Data Processing and Analysis Consortium (DPAC, http://www. cosmos.esa.int/web/gaia/dpac/consortium). Funding for the DPAC has been provided by national institutions, in particular the institutions participating in the Gaia Multilateral Agreement. This research has made use of the SIMBAD database and the VizieR catalogue access tool operated at CDS, Strasbourg, France. The original description of the VizieR service was published in Ochsenbein et al. (2000).

\section{References}

Acharova, I. A., Mishurov, Y. N., \& Kovtyukh, V. V. 2012, MNRAS, 420, 1590 Anderson, R. I. 2018, A\&A, 611, L7

Anderson, R. I., Saio, H., Ekström, S., Georgy, C., \& Meynet, G. 2016, A\&A, 591, A8

Arenou, F., Grenon, M., \& Gomez, A. 1992, A\&A, 258, 104

Arenou, F., Luri, X., Babusiaux, C., et al. 2018, A\&A, 616, A17

Barnes, III, T. G., Fernley, J. A., Frueh, M. L., et al. 1997, PASP, 109, 645

Benedict, G. F., McArthur, B. E., Feast, M. W., et al. 2007, AJ, 133, 1810

Berdnikov, L. N., Dambis, A. K., \& Vozyakova, O. V. 2000, A\&AS, 143, 211

Bond, H. E., Nelan, E. P., Remage Evans, N., Schaefer, G. H., \& Harmer, D. 2018, ApJ, 853, 55

Caldwell, J. A. R., \& Coulson, I. M. 1987, AJ, 93, 1090

Cardelli, J. A., Clayton, G. C., \& Mathis, J. S. 1989, ApJ, 345, 245

Carpenter, J. M. 2001, AJ, 121, 2851

Casertano, S., Riess, A. G., Anderson, J., et al. 2016, ApJ, 825, 11

Clementini, G., Ripepi, V., Molinaro, R., et al. 2018, A\&A, submitted, [arXiv:1805.02079]

de Grijs, R., Wicker, J. E., \& Bono, G. 2014, AJ, 147, 122

Drimmel, R., Cabrera-Lavers, A., \& López-Corredoira, M. 2003, A\&A, 409, 205

ESA 1997, VizieR Online Data Catalog: I/239

Evans, N. R. 1994, ApJ, 436, 273

Evans, N. R. 2000, AJ, 119, 3050

Evans, N. R., Schaefer, G. H., Bond, H. E., et al. 2008, AJ, 136, 1137

Evans, N. R., Bond, H. E., Schaefer, G. H., et al. 2016, AJ, 151, 129

Feast, M. 1999, PASP, 111, 775

Feast, M. W., \& Catchpole, R. M. 1997, MNRAS, 286, L1

Feast, M. W., Laney, C. D., Kinman, T. D., van Leeuwen, F., \& Whitelock, P. A. 2008, MNRAS, 386, 2115

Fernie, J. D., Evans, N. R., Beattie, B., \& Seager, S. 1995, IBVS, 4148

Fouqué, P., Arriagada, P., Storm, J., et al. 2007, A\&A, 476, 73

Gaia Collaboration (Brown, A. G. A., et al.) 2016a, A\&A, 595, A2

Gaia Collaboration (Prusti, T., et al.) 2016b, A\&A, 595, A1

Gaia Collaboration (Clementini, G., et al. ) 2017, A\&A, 605, A79

Gaia Collaboration (Brown, A. G. A.) 2018, A\&A, 616, A1

Gallenne, A., Monnier, J. D., Mérand, A., et al. 2013, A\&A, 552, A21

Gehrz, R. D., \& Hackwell, J. A. 1974a, ApJ, 193, 385

Gehrz, R. D., Hackwell, J. A., \& Jones, T. W. 1974b, ApJ, 191, 675

Genovali, K., Lemasle, B., Bono, G., et al. 2014, A\&A, 566, A37

Genovali, K., Lemasle, B., da Silva, R., et al. 2015, A\&A, 580, A17

Groenewegen, M. A. T. 2008, A\&A, 488, 935

Groenewegen, M. A. T. 2013, A\&A, 550, A70

Holl, B., Audard, M., Nienartowicz, K., et al. 2018, A\&A, 618, A30
Inno, L., Bono, G., Matsunaga, N., et al. 2016, ApJ, 832, 176

Jacyszyn-Dobrzeniecka, A. M., Skowron, D. M., Mróz, P., et al. 2016, Acta Astron., 66, 149

Kashuba, S. V., Andrievsky, S. M., Chekhonadskikh, F. A., et al. 2016, MNRAS, 461,839

Kervella, P., Bond, H. E., Cracraft, M., et al. 2014, A\&A, 572, A7

Klagyivik, P., \& Szabados, L. 2009, A\&A, 504, 959

Koen, C., \& Laney, D. 1998, MNRAS, 301, 582

Koen, C., Marang, F., Kilkenny, D., \& Jacobs, C. 2007, MNRAS, 380, 1433

Laney, C. D., \& Caldwell, J. A. R. 2007, MNRAS, 377, 147

Laney, C. D., \& Stobie, R. S. 1992, A\&AS, 93, 93

Lanoix, P., Paturel, G., \& Garnier, R. 1999, MNRAS, 308, 969

Lemasle, B., Groenewegen, M. A. T., Grebel, E. K., et al. 2017, A\&A, 608, A85

Lenz, P., \& Breger, M. 2005, Commun. Asteroseismology, 146, 53

Lindegren, L., Hernandez, J., Bombrun, A., et al. 2018, A\&A, 616, A2

Luck, R. E., \& Lambert, D. L. 2011, AJ, 142, 136

Luck, R. E., Moffett, T. J., Barnes, III, T. G., \& Gieren, W. P. 1998, AJ, 115, 605

Luri, X., Brown, A. G. A., Sarro, L. M., et al. 2018, A\&A, 616, A9

Lutz, T. E., \& Kelker, D. H. 1973, PASP, 85, 573

Marshall, D. J., Robin, A. C., Reylé, C., Schultheis, M., \& Picaud, S. 2006, A\&A, 453, 635

Martin, R. P., Andrievsky, S. M., Kovtyukh, V. V., et al. 2015, MNRAS, 449, 4071

McGonegal, R., McAlary, C. W., McLaren, R. A., \& Madore, B. F. 1983, ApJ, 269, 641

Mel'nik, A. M., Rautiainen, P., Berdnikov, L. N., Dambis, A. K., \& Rastorguev, A. S. 2015, Astron. Nachr., 336, 70

Mérand, A., Kervella, P., Coudé du Foresto, V., et al. 2006, A\&A, 453, 155

Molnár, L., Derekas, A., Szabó, R., et al. 2017, MNRAS, 466, 4009

Monson, A. J., \& Pierce, M. J. 2011, ApJS, 193, 12

Muraveva, T., Delgado, H. E., Clementini, G., Sarro, L. M., \& Garofalo, A. 2018 , MNRAS, 481, 1195

Ngeow, C.-C. 2012, ApJ, 747, 50

Ngeow, C.-C., Kanbur, S. M., Neilson, H. R., Nanthakumar, A., \& Buonaccorsi, J. 2009, ApJ, 693, 691

Ngeow, C.-C., Kanbur, S. M., Bhardwaj, A., \& Singh, H. P. 2015, ApJ, 808, 67

Ochsenbein, F., Bauer, P., \& Marcout, J. 2000, A\&AS, 143, 23

Pietrzyński, G., Graczyk, D., Gieren, W., et al. 2013, Nature, 495, 76

Press, W., Teukolsky, S., Vetterling, W., \& Flannery, B. 1992, in Numerical Recipes in C (Cambridge: Cambridge University Press)

Proxauf, B., da Silva, R., Kovtyukh, V. V., et al. 2018, A\&A, 616, A82

Riess, A. G., Casertano, S., Anderson, J., MacKenty, J., \& Filippenko, A. V. 2014, ApJ, 785, 161

Riess, A. G., Macri, L. M., Hoffmann, S. L., et al. 2016, ApJ, 826, 56

Riess, A. G., Casertano, S., Yuan, W., et al. 2018a, ApJ, 855, 136

Riess, A. G., Casertano, S., Yuan, W., et al. 2018b, ApJ, 861, 126

Ripepi, V., Moretti, M. I., Marconi, M., et al. 2012, MNRAS, 424, 1807

Ripepi, V., Cioni, M.-R. L., Moretti, M. I., et al. 2017, MNRAS, 472, 808

Romaniello, M., Primas, F., Mottini, M., et al. 2008, A\&A, 488, 731

Schechter, P. L., Avruch, I. M., Caldwell, J. A. R., \& Keane, M. J. 1992, AJ, 104, 1930

Smith, B. J., Price, S. D., \& Baker, R. I. 2004, ApJS, 154, 673

Stassun, K. G., \& Torres, G. 2018, ApJ, 862, 61

Storm, J., Gieren, W., Fouqué, P., et al. 2011a, A\&A, 534, A94

Storm, J., Gieren, W., Fouqué, P., et al. 2011b, A\&A, 534, A95

Sziládi, K., Vinkó, J., Poretti, E., Szabados, L., \& Kun, M. 2007, A\&A, 473, 579

Tammann, G. A., Sandage, A., \& Reindl, B. 2003, A\&A, 404, 423

Turner, D. G. 2010, Ap\&SS, 326, 219

Turner, D. G., Kovtyukh, V. V., Usenko, I. A., \& Gorlova, N. I. 2013, ApJ, 762, L8

van Leeuwen, F. 2007, A\&A, 474, 653

van Leeuwen, F. 2008, VizieR Online Data Catalog: I/311

van Leeuwen, F. 2013, A\&A, 550, L3

van Leeuwen, F., Feast, M. W., Whitelock, P. A., \& Laney, C. D. 2007, MNRAS, 379,723

Watson, C. L., Henden, A. A., \& Price, A. 2006, Society for Astronomical Sciences Annual Symposium, 25, 47

Welch, D. L., Wieland, F., McAlary, C. W., et al. 1984, ApJS, 54, 547

Zinn, J. C., Pinsonneault, M. H., Huber, D., \& Stello, D. 2018, ApJ, submitted, [arXiv:1805.02650] 


\section{Appendix A: Initial run of models}

Table A.1 shows the results of fitting period-luminosity relations in the $V, K$, and $W V K$ bands based on several selection criteria, which include the goodness of fit, fundamental or overtone pulsators, period cuts, and parallax zero-point offsets. This initial set of models was calculated to investigate the parameter space and arrive at a "best" sample, which is used in the main text.

The table is organised as follows. The models are grouped by the parallax that was used, GDR2-only or GDR2+External values, as outlined in Sect. 6 , and by filter $(V, K$, or $W V K)$. Then the solutions are given applying the various constraints listed in Col. 4.
Inspecting the results shows that the overtone pulsators (using their fundamentalised period) have significantly different $P L$ relations than the FU mode pulsators. This was not investigated further, but led to the conservative choice of using only FU mode Cepheids in the final sample.

A cut on period was investigated and finally implemented (period between 2.7 and 35 days). The exact values are less important, but they were used to avoid a possible contamination by unrecognised overtone pulsators (at the short period end) and to make a fairer comparison to samples in the Magellanic Clouds where such period cuts are typically used.

Stars for the final sample were selected to have $|\mathrm{GOF}|<8$ and no excess noise. This is related to the problem of very poor astrometric solutions, which can occur for the brightest stars, but also due to binarity (see main text). 
Table A.1. $P L$ relations of the form $M=\alpha+\beta \log P$.

\begin{tabular}{|c|c|c|c|}
\hline$\alpha$ & $\beta$ & $N$ & Constraints \\
\hline \multicolumn{4}{|c|}{ GDR2 parallaxes } \\
\hline & \multicolumn{3}{|c|}{$V$ band } \\
\hline$-1.984 \pm 0.121$ & $-2.326 \pm 0.115$ & 425 & all \\
\hline$-1.985 \pm 0.136$ & $-2.321 \pm 0.134$ & 419 & $|\mathrm{GOF}|<100$ \\
\hline$-1.956 \pm 0.043$ & $-2.337 \pm 0.049$ & 376 & $|\mathrm{GOF}|<20$ \\
\hline$-2.033 \pm 0.051$ & $-2.220 \pm 0.061$ & 293 & $|\mathrm{GOF}|<10$ \\
\hline$-2.049 \pm 0.055$ & $-2.201 \pm 0.066$ & 250 & $|\mathrm{GOF}|<8$ \\
\hline$-2.138 \pm 0.074$ & $-2.084 \pm 0.092$ & 197 & $|\mathrm{GOF}|<6$ \\
\hline$-2.134 \pm 0.090$ & $-2.093 \pm 0.110$ & 113 & $|\mathrm{GOF}|<4$ \\
\hline$-2.047 \pm 0.055$ & $-2.202 \pm 0.067$ & 248 & $\begin{array}{l}|\mathrm{GOF}|<8, \epsilon_{i}<0.001 \\
|\mathrm{GOF}|<8, \epsilon_{i}<0.001, \text { and } \ldots\end{array}$ \\
\hline$-1.881 \pm 0.057$ & $-2.416 \pm 0.065$ & 211 & FU \\
\hline$-2.661 \pm 0.176$ & $-1.344 \pm 0.247$ & 37 & FO \\
\hline$-1.852 \pm 0.060$ & $-2.446 \pm 0.068$ & 205 & FU, $P>2.5 \mathrm{~d}$ \\
\hline$-1.857 \pm 0.060$ & $-2.441 \pm 0.068$ & 200 & FU, $P>3.0 \mathrm{~d}$ \\
\hline$-1.869 \pm 0.059$ & $-2.431 \pm 0.070$ & 185 & FU, $P>3.5 \mathrm{~d}$ \\
\hline$-1.911 \pm 0.080$ & $-2.393 \pm 0.093$ & 159 & FU, $P>2.5 \mathrm{~d},|\mathrm{GOF}|<6$ \\
\hline$-1.882 \pm 0.115$ & $-2.363 \pm 0.131$ & 90 & FU, $P>2.5 \mathrm{~d},|\mathrm{GOF}|<4$ \\
\hline$-1.847 \pm 0.072$ & $-2.457 \pm 0.086$ & 202 & FU, $P>2.5 \mathrm{~d}, P<35$ \\
\hline$-1.886 \pm 0.060$ & $-2.415 \pm 0.068$ & 207 & FU, no uncertain types \\
\hline$-1.794 \pm 0.058$ & $-2.385 \pm 0.066$ & 205 & FU, $P>2.5 \mathrm{~d}, \mathrm{ZPoff}=-0.029 \mathrm{mas}$ \\
\hline$-1.759 \pm 0.057$ & $-2.353 \pm 0.065$ & 205 & FU, $P>2.5 \mathrm{~d}$, ZPoff $=-0.046$ mas \\
\hline$-1.883 \pm 0.113$ & $-2.443 \pm 0.126$ & 205 & FU, $P>2.5 \mathrm{~d}, \delta_{\mathrm{PL}}=0.20$ \\
\hline \multicolumn{4}{|c|}{$K$ band } \\
\hline$-2.980 \pm 0.031$ & $-3.060 \pm 0.038$ & 425 & all \\
\hline$-2.985 \pm 0.029$ & $-3.051 \pm 0.035$ & 419 & $|\mathrm{GOF}|<100$ \\
\hline$-3.008 \pm 0.031$ & $-3.023 \pm 0.036$ & 376 & $|\mathrm{GOF}|<20$ \\
\hline$-3.073 \pm 0.036$ & $-2.929 \pm 0.045$ & 294 & $|\mathrm{GOF}|<10$ \\
\hline$-3.083 \pm 0.039$ & $-2.907 \pm 0.048$ & 250 & $|\mathrm{GOF}|<8$ \\
\hline$-3.171 \pm 0.053$ & $-2.787 \pm 0.067$ & 197 & $|\mathrm{GOF}|<6$ \\
\hline$-3.209 \pm 0.063$ & $-2.769 \pm 0.081$ & 113 & $|\mathrm{GOF}|<4$ \\
\hline$-3.081 \pm 0.039$ & $-2.907 \pm 0.045$ & 248 & $\begin{array}{l}|\mathrm{GOF}|<8, \epsilon_{i}<0.001 \\
|\mathrm{GOF}|<8, \epsilon_{i}<0.001, \text { and }\end{array}$ \\
\hline$-2.985 \pm 0.041$ & $-3.083 \pm 0.048$ & 211 & FU \\
\hline$-3.622 \pm 0.125$ & $-1.974 \pm 0.192$ & 37 & FO \\
\hline$-2.953 \pm 0.044$ & $-3.119 \pm 0.051$ & 205 & FU, $P>2.5 \mathrm{~d}$ \\
\hline$-2.964 \pm 0.044$ & $-3.107 \pm 0.052$ & 200 & FU, $P>3.0 \mathrm{~d}$ \\
\hline$-2.992 \pm 0.052$ & $-3.079 \pm 0.060$ & 185 & FU, $P>3.5 \mathrm{~d}$ \\
\hline$-3.017 \pm 0.057$ & $-3.053 \pm 0.069$ & 159 & FU, $P>2.5 \mathrm{~d},|\mathrm{GOF}|<6$ \\
\hline$-3.146 \pm 0.075$ & $-2.869 \pm 0.094$ & 90 & FU, $P>2.5 \mathrm{~d},|\mathrm{GOF}|<4$ \\
\hline$-3.181 \pm 0.091$ & $-2.829 \pm 0.107$ & 76 & FU, $P>3.5 \mathrm{~d},|\mathrm{GOF}|<4$ \\
\hline$-2.957 \pm 0.044$ & $-3.117 \pm 0.053$ & 201 & FU, $P>2.5 \mathrm{~d}$, no uncertain \\
\hline$-2.918 \pm 0.047$ & $-3.166 \pm 0.060$ & 202 & FU, $P>2.5 \mathrm{~d}, P<35$ \\
\hline$-2.894 \pm 0.042$ & $-3.055 \pm 0.048$ & 205 & FU, $P>2.5 \mathrm{~d}, \mathrm{ZPoff}=-0.029 \mathrm{mas}$ \\
\hline$-2.861 \pm 0.040$ & $-3.021 \pm 0.047$ & 205 & FU, $P>2.5 \mathrm{~d}$, ZPoff $=-0.046 \mathrm{mas}$ \\
\hline$-2.957 \pm 0.053$ & $-3.114 \pm 0.061$ & 205 & FU, $P>2.5 \mathrm{~d}, \delta_{\mathrm{PL}}=0.066$ \\
\hline \multicolumn{4}{|c|}{$W V K$ band } \\
\hline$-3.114 \pm 0.031$ & $-3.158 \pm 0.038$ & 425 & all \\
\hline$-3.118 \pm 0.030$ & $-3.149 \pm 0.036$ & 419 & $|\mathrm{GOF}|<100$ \\
\hline$-3.149 \pm 0.031$ & $-3.113 \pm 0.037$ & 376 & $|\mathrm{GOF}|<20$ \\
\hline$-3.213 \pm 0.035$ & $-3.020 \pm 0.045$ & 293 & $|\mathrm{GOF}|<10$ \\
\hline$-3.221 \pm 0.038$ & $-2.998 \pm 0.048$ & 250 & $|\mathrm{GOF}|<8$ \\
\hline$-3.311 \pm 0.052$ & $-2.876 \pm 0.068$ & 197 & $|\mathrm{GOF}|<6$ \\
\hline$-3.360 \pm 0.063$ & $-2.849 \pm 0.082$ & 113 & $|\mathrm{GOF}|<4$ \\
\hline$-3.221 \pm 0.038$ & $-2.998 \pm 0.045$ & 248 & $|\mathrm{GOF}|<8, \epsilon_{i}<0.001$ \\
\hline
\end{tabular}

Notes. $N$ is the number of stars in the solution. 
Table A.1. continued.

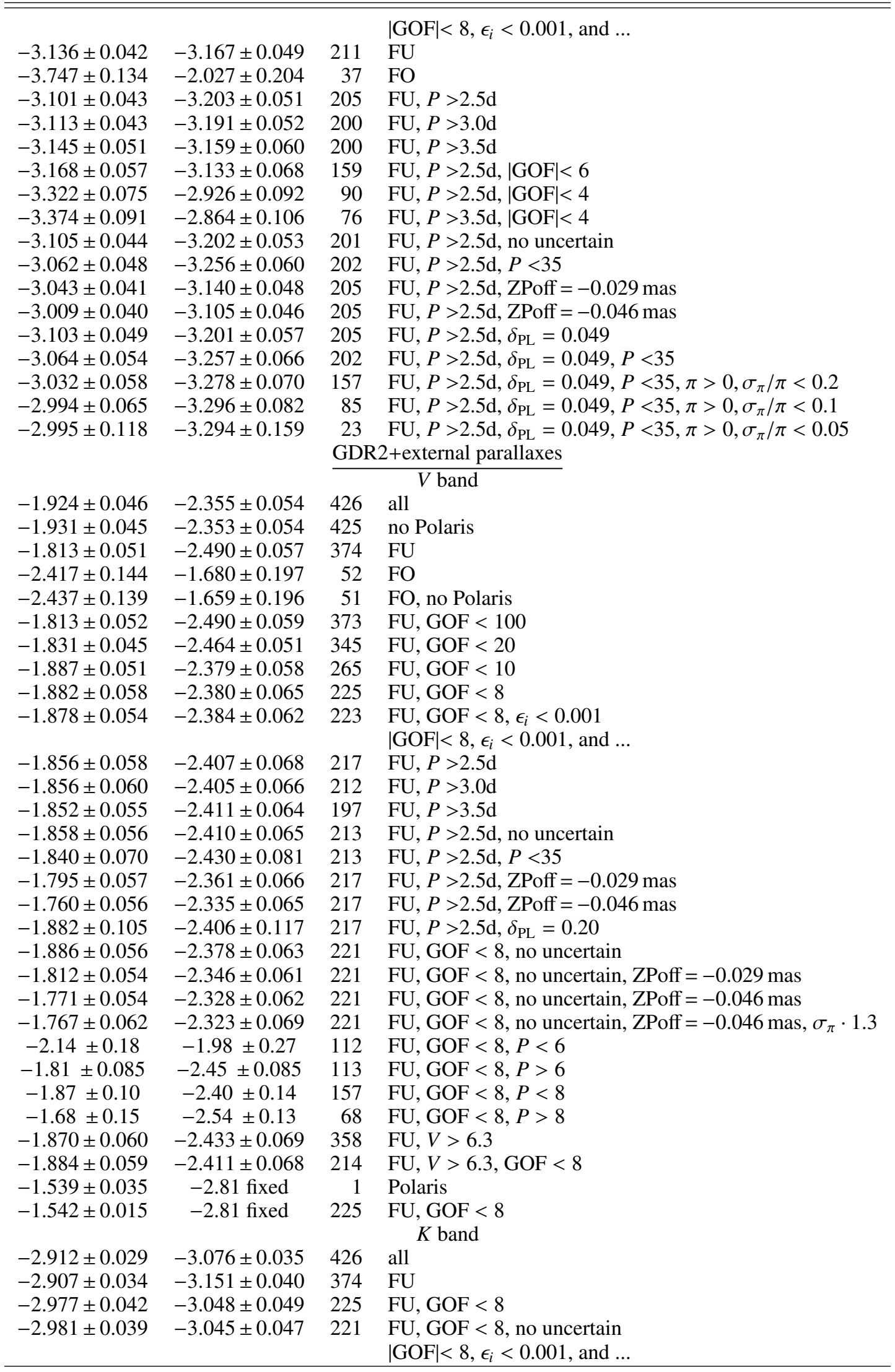


Table A.1. continued.

\begin{tabular}{|c|c|c|c|}
\hline$-2.943 \pm 0.042$ & $-3.084 \pm 0.051$ & 217 & $\mathrm{FU}, \mathrm{GOF}<8, P>2.5 \mathrm{~d}$ \\
\hline$-2.950 \pm 0.042$ & $-3.075 \pm 0.048$ & 212 & FU, GOF $<8, P>3.0 \mathrm{~d}$ \\
\hline$-2.957 \pm 0.049$ & $-3.069 \pm 0.056$ & 197 & $\mathrm{FU}, \mathrm{GOF}<8, P>3.5 \mathrm{~d}$ \\
\hline$-2.911 \pm 0.048$ & $-3.130 \pm 0.058$ & 213 & FU, GOF $<8, P>2.5, P<35 \mathrm{~d}$ \\
\hline$-2.882 \pm 0.040$ & $-3.037 \pm 0.048$ & 217 & $\mathrm{FU}, \mathrm{GOF}<8, P>2.5 \mathrm{~d}, \mathrm{ZPoff}=-0.029 \mathrm{mas}$ \\
\hline$-2.848 \pm 0.039$ & $-3.011 \pm 0.047$ & 217 & FU, GOF $<8, P>2.5 \mathrm{~d}, \mathrm{ZPoff}=-0.046 \mathrm{mas}$ \\
\hline$-2.946 \pm 0.052$ & $-3.084 \pm 0.060$ & 217 & $\mathrm{FU}, \mathrm{GOF}<8, P>2.5 \mathrm{~d}, \delta_{\mathrm{PL}}=0.066$ \\
\hline$-2.976 \pm 0.042$ & $-3.051 \pm 0.050$ & 220 & FU, GOF $<8$, no uncertain, $P>2 \mathrm{~d}$ \\
\hline$-2.956 \pm 0.042$ & $-3.074 \pm 0.051$ & 209 & FU, GOF $<8$, no uncertain, $P>3 \mathrm{~d}$ \\
\hline$-2.959 \pm 0.050$ & $-3.070 \pm 0.058$ & 180 & FU, GOF $<8$, no uncertain, $P>4 \mathrm{~d}$ \\
\hline$-2.805 \pm 0.061$ & $-3.214 \pm 0.066$ & 140 & FU, GOF $<8$, no uncertain, $P>5 \mathrm{~d}$ \\
\hline$-2.915 \pm 0.076$ & $-3.119 \pm 0.077$ & 110 & FU, GOF $<8$, no uncertain, $P>6 \mathrm{~d}$ \\
\hline$-2.917 \pm 0.071$ & $-3.012 \pm 0.072$ & 110 & FU, GOF $<8$, no uncertain, $P>6 \mathrm{~d}$, ZPoff $=-0.029$ mas \\
\hline$-2.915 \pm 0.071$ & $-2.956 \pm 0.070$ & 110 & FU, GOF $<8$, no uncertain, $P>6 \mathrm{~d}$, ZPoff $=-0.046$ mas \\
\hline$-2.897 \pm 0.095$ & $-3.111 \pm 0.096$ & 110 & FU, GOF $<8$, no uncertain, $P>6 \mathrm{~d}$, ZPoff $=-0.000$ mas, $\sigma_{\pi} \cdot 1.3$ \\
\hline$-2.765 \pm 0.008$ & -3.26 fixed & 426 & all \\
\hline$-2.484 \pm 0.028$ & -3.26 fixed & 1 & Polaris \\
\hline$-2.735 \pm 0.010$ & -3.26 fixed & 269 & GOF $<8$ \\
\hline$-2.807 \pm 0.010$ & -3.26 fixed & 225 & $\begin{array}{r}\mathrm{FU}, \mathrm{GOF}<8 \\
W V K \text { band }\end{array}$ \\
\hline$-3.045 \pm 0.030$ & $-3.174 \pm 0.035$ & 426 & all \\
\hline$-3.122 \pm 0.036$ & $-3.026 \pm 0.045$ & 269 & GOF $<8$ \\
\hline$-3.125 \pm 0.042$ & $-3.133 \pm 0.048$ & 225 & $\mathrm{GOF}<8, \mathrm{FU}$ \\
\hline$-3.609 \pm 0.042$ & $-2.093 \pm 0.200$ & 44 & $\mathrm{GOF}<8, \mathrm{FO}$ \\
\hline$-3.092 \pm 0.042$ & $-3.166 \pm 0.050$ & 218 & $\mathrm{GOF}<8, \mathrm{FU}, P>2.5 \mathrm{~d}$ \\
\hline$-3.099 \pm 0.041$ & $-3.161 \pm 0.047$ & 213 & $\mathrm{GOF}<8, \mathrm{FU}, P>3.0 \mathrm{~d}$ \\
\hline$-3.108 \pm 0.048$ & $-3.151 \pm 0.055$ & 197 & $\mathrm{GOF}<8, \mathrm{FU}, P>3.5 \mathrm{~d}$ \\
\hline$-3.060 \pm 0.048$ & $-3.210 \pm 0.060$ & 214 & $\mathrm{GOF}<8, \mathrm{FU}, P>2.5, P<35 \mathrm{~d}$ \\
\hline$-3.031 \pm 0.041$ & $-3.119 \pm 0.048$ & 218 & $\mathrm{GOF}<8, \mathrm{FU}, P>2.5 \mathrm{~d}, \mathrm{ZPoff}=-0.029 \mathrm{mas}$ \\
\hline$-2.996 \pm 0.040$ & $-3.093 \pm 0.048$ & 218 & $\mathrm{GOF}<8, \mathrm{FU}, P>2.5 \mathrm{~d}, \mathrm{ZPoff}=-0.046 \mathrm{mas}$ \\
\hline$-3.095 \pm 0.047$ & $-3.166 \pm 0.053$ & 218 & $\mathrm{GOF}<8, \mathrm{FU}, P>2.5 \mathrm{~d}, \delta_{\mathrm{PL}}=0.049$ \\
\hline
\end{tabular}

\section{Appendix B: The Polaris system}

In this appendix we discuss the distance to the Polaris system, the CC $\alpha$ UMi (Polaris $\mathrm{A}^{4}$ ) and its companion Polaris B, located at about $18^{\prime \prime}$. Polaris A is listed in GDR2 with the largest GOF of the CCs in the sample and without parallax. The parallax used is the average of the available HIPPARCOS parallaxes (Table 3), but as FO pulsators are excluded in the final analysis, Polaris A is not actually used in this paper. Polaris B is listed in GDR2 with a parallax of $7.292 \pm 0.028$ mas. The astrometric solution has zero excess noise and a GOF value of about 12 , which means it would not make it through the selection criterion of GOF $<8$ that was applied to the CCs in the sample.

Polaris B has a recent HST based parallax of $6.26 \pm$ 0.26 mas (Bond et al. 2018) which differs by $4 \sigma$ from the GDR2 value. Anderson (2018) showed that adopting the parallax by Bond et al. (2018) for Polaris A a consistent picture emerged of Polaris as a $7 M_{\odot}$ first-overtone CC near the hot boundary of the first IS crossing. This conclusion was based on the Geneva set of rotating stellar evolutionary models (Anderson et al. 2016) and considering various constraints (the rate of period change, the Wesenheit WVI PL relation, the location in the $M_{V}-(B-V)$ colour-magnitude diagram, the interferometrically determined radius, spectroscopic N/C and N/O abundance ratios, and a dy-

\footnotetext{
4 Or Aa to be precise, as this is a binary system itself, with a companion that is of order 5 mag fainter at less than $0.2^{\prime \prime}$ distance (Evans et al. 2008).
}

namical mass measurement). Anderson (2018) does not discuss the quality of the model that can be obtained using the larger HIPPARCOS parallax ${ }^{5}$.

Based on the current best estimates of the $P L$ relations (solutions 200-202 in Table 8) the photometric parallax of Polaris A is predicted using Monte Carlo simulations, that is, taking into account the uncertainty in $V$ and $K$ photometry and reddening value, and the width of the instability strip. A fundamentalised period of 5.673 days is used. The results are listed in Table B.1.

For the standard case the predicted parallax distribution based on the $W V K$ relation gives a median and dispersion (based on $1.483 \times$ the median absolute deviation, which is equivalent to $\sigma$ in the case of a Gaussian distribution) of $6.99 \pm 0.19$ mas. The $K$ band gives a consistent result of $7.12 \pm 0.23$ mas; the $V$ band also gives a consistent, but more uncertain, value of $8.1 \pm 0.8$ mas. Figure B. 1 shows the distribution in predicted parallax based on the $W V K P L$ relation and compares it to the various estimates in the literature. These parallax estimates depend on the assumed parallax zero-point offset, and Table B.1 list values for some other offsets for the Wesenheit $W V K$ magnitude only, as it gives the best precision.

The calculations and the resulting parallax distribution have assumed so far that Polaris A is located in the centre of the IS with the width of the IS represented by a Gaussian with width $\delta_{\mathrm{PL}}=0.049 \mathrm{mag}$. Anderson (2018) have argued that Polaris A

\footnotetext{
5 His paper appeared pre-GDR2.
} 
Table B.1. Photometric parallax predictions for Polaris.

\begin{tabular}{cl}
\hline \hline $\begin{array}{c}\text { Parallax } \\
(\mathrm{mas})\end{array}$ & Remarks \\
\hline $6.989 \pm 0.186$ & $W V K($ Sol. 200 from Table 8$)$ \\
$7.122 \pm 0.234$ & $K$ (Sol. 201) \\
$8.080 \pm 0.759$ & $V$ (Sol. 202) \\
$6.484 \pm 0.174$ & $W V K$, ZPoff $=0.0$ mas \\
$6.782 \pm 0.181$ & $W V K$, ZPoff $=-0.029$ mas \\
$6.989 \pm 0.186$ & $W V K$, ZPoff $=-0.049$ mas (Sol. 200) \\
$7.103 \pm 0.189$ & $W V K$, ZPoff $=-0.060$ mas \\
$7.310 \pm 0.194$ & $W V K$, ZPoff $=-0.080$ mas \\
$7.515 \pm 0.198$ & $W V K$, ZPoff $=-0.100$ mas \\
$6.687 \pm 0.102$ & $W V K$, ZPoff $=-0.049$ mas, location in the IS, $W V K+0.1 \mathrm{mag}, \delta_{\mathrm{PL}}=0 \mathrm{mag}$ \\
$6.931 \pm 0.185$ & $W V K$, ZPoff $=-0.049$ mas, NIR excess, $\Delta K=0.016 \pm 0.004 \mathrm{mag}$ \\
$7.256 \pm 0.194$ & $W V K$, ZPoff $=-0.049$ mas, alternative $K$ mag, $K=0.58 \pm 0.028 \mathrm{mag}$ \\
\hline
\end{tabular}

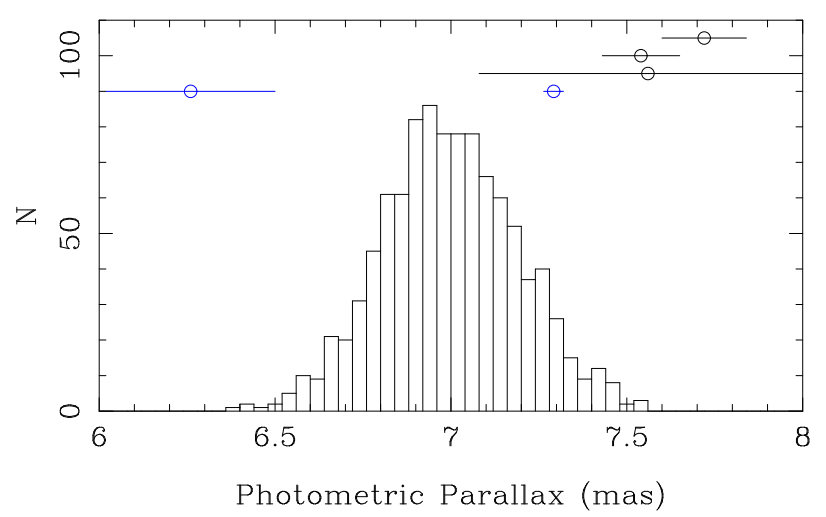

Fig. B.1. Distribution of the photometric parallax predicted for Polaris based on the $W V K P L$ relation. The blue lines give the parallax and error determined for Polaris B (6.3 mas from Bond et al. 2018, 7.3 mas from GDR2) and the black lines the various estimates from HIPPARCOS for Polaris A (see Table 3).

is located at the blue edge (i.e. the hotter end) of the IS (based on the shorter parallax by Bond et al. 2018). Adding +0.1 mag to the $W V K$ colour of Polaris A to simulate the colour it would have at the centre of the IS results in a parallax estimate of $6.69 \pm$ 0.10 mas (assuming $\delta_{\mathrm{PL}}=0$ mag now).

A particular problem for Polaris A is that its $K$-band magnitude is not very well established, and that interferometric observations have revealed a small excess of $0.016 \pm 0.004$ mag in $K$ (Mérand et al. 2006) due to the presence of an extended circumstellar envelope. Making the standard adopted value (see the main text) of $K=0.652 \pm 0.028$ mag fainter by this amount (and adding the error in quadrature) has a small effect. The actual value has a potentially larger impact. van Leeuwen et al. (2007) also use the COBE-DIRBE flux of Polaris, but adopting a different calibration scheme and magnitudes for Procyon arrive at $J=0.98 \mathrm{mag}$ and $K=0.60 \mathrm{mag}$ on the SAAO system (with no error bars given). Using the transformation formulae in Carpenter (2001), we arrive at $K=0.58 \mathrm{mag}$ on the 2MASS system. Using this value (with the same error bar of 0.028 mag as we derived) results in a significantly larger parallax.

In summary, the predicted photometric parallax is not conclusive. It is roughly halfway between the HIPPARCOS parallax estimates for Polaris A and the GDR2 estimate for Polaris B, and the estimate by Bond et al. (2018). 